\title{
Medium-Frequency Transformer Scaling Laws: Derivation, Verification, and Critical Analysis
}

\author{
Thomas Guillod and Johann Walter Kolar
}

\begin{abstract}
Medium-frequency transformers (MFTs) are one of the fundamental building blocks of modern power electronic converters. The usage of increased frequencies leads to improved characteristics, i.e., efficiency and power density (volumetric and gravimetric) but also to design challenges and constraints. This paper reviews the analytical modeling of MFTs. More particularly, the mapping between the design space and the performance space is analyzed. It is found that wide regions of the design space are mapped to a narrow region in the performance space, i.e., the optimum is flat and designs with very different parameters features similar performances (design space diversity). Scaling laws are derived for optimal MFTs operated at different power ratings and power densities, which provide a comprehensive and general insight on the achievable performances. In a next step, the results obtained with the analytical model are compared to numerical simulations. It is concluded that the derived scaling laws capture qualitatively and quantitatively the behavior of MFTs, but should be used with caution for accurate design processes.
\end{abstract}

Index Terms-Analytical model, medium-frequency transformers, numerical simulations, optimization, power converters, power electronics, pareto front, scaling laws.

\section{NOMENCLATURE}

$$
A_{\mathrm{c}}
$$$$
t_{\mathrm{c}}
$$$$
z_{\mathrm{c}}
$$

$V_{\mathrm{c}}$

$A_{\text {w }}$

$d_{\mathrm{w}}$

$h_{\mathrm{w}}$

$V_{\mathrm{w}}$

$x_{\mathrm{cw}}$

$x_{\mathrm{c}}$

$x_{\mathrm{w}}$

$A_{\mathrm{t}}$

$V_{\text {box }}$

$m_{\text {tot }}$

$n$

Core cross section.
Core limb width.
Core depth.
Core volume.
Winding window cross section.
Winding window width.
Winding window height.
Winding volume.
Ratio between $A_{\mathrm{c}}$ and $A_{\mathrm{w}}$.
Ratio between $z_{\mathrm{c}}$ and $2 t_{\mathrm{c}}$.
Ratio between $h_{\mathrm{w}}$ and $d_{\mathrm{w}}$.
Exposed area for cooling.
MFT boxed volume.
MFT mass.
Number of turns (both windings).

Manuscript received August 8, 2019; revised October 14, 2019; accepted November 21, 2019. Date of publication March 31, 2020; date of current version February 10, 2020. (Corresponding author: Thomas Guillod.)

All authors are with the Power Electronic Systems Laboratory, ETH Zurich, Switzerland (e-mail: guillod@lem.ee.ethz.ch).

Digital Object Identifier 10.24295/CPSSTPEA.2020.00003

\begin{tabular}{|c|c|}
\hline$\rho$ & Volumetric power density. \\
\hline$\gamma$ & Gravimetric power density. \\
\hline$P$ & MFT active rated power. \\
\hline$S$ & MFT apparent power. \\
\hline $\cos (\varphi)$ & MFT power factor. \\
\hline$f$ & MFT operating frequency. \\
\hline$I_{\mathrm{RMS}}$ & MFT RMS current. \\
\hline$V_{\mathrm{RMS}}$ & MFT RMS voltage. \\
\hline$B_{\mathrm{pk}}$ & Core peak flux density. \\
\hline$k_{\mathrm{c}} / \alpha_{\mathrm{c}} / \beta_{\mathrm{c}}$ & Core Steinmetz parameters (empirical). \\
\hline$f_{\mathrm{c}, \max }$ & Max. frequency for the core. \\
\hline$B_{\text {sat }}$ & Core saturation flux density. \\
\hline$p_{\mathrm{c}}$ & Core loss density. \\
\hline$P_{\mathrm{c}}$ & Core losses. \\
\hline$\mu_{0}$ & Vacuum permeability. \\
\hline$k_{\mathrm{w}}$ & Winding fill factor. \\
\hline$J_{\text {RMS }}$ & Winding RMS current density. \\
\hline$d_{\mathrm{s}}$ & Winding strand diameter. \\
\hline$\sigma$ & Winding copper conductivity. \\
\hline$a_{\mathrm{w}}$ & Proximity effect factor. \\
\hline$\delta$ & Skin depth . \\
\hline$p_{\mathrm{w}}$ & Winding loss density. \\
\hline$P_{\mathrm{w}}$ & Winding losses. \\
\hline$J_{\mathrm{RMS}, \max }$ & Max. winding RMS current density. \\
\hline$h_{\mathrm{t}}$ & Surface convection coefficient. \\
\hline$k_{\mathrm{t}} / v_{\mathrm{t}} / \kappa_{\mathrm{t}}$ & Convection parameters (empirical). \\
\hline$\Delta T$ & Temperature rise (with respect to amb.) \\
\hline$\Delta T_{\max }$ & Max. allowable temperature rise. \\
\hline$r_{\mathrm{cw}}$ & Ratio between $P_{\mathrm{c}}$ and $P_{\mathrm{w}}$. \\
\hline$r_{\mathrm{w}}$ & $\mathrm{AC} / \mathrm{DC}$ winding resistance ratio. \\
\hline$P_{1}$ & Total losses (core and windings). \\
\hline$\eta_{\mathrm{pl}}$ & MFT efficiency (50\% load). \\
\hline$\eta_{\mathrm{fl}}$ & MFT efficiency (100\% load). \\
\hline & I. INTRODUCTION \\
\hline
\end{tabular}

T OW-FREQUENCY transformers (LFTs) are widely Lused for voltage transformation/adaptation and galvanic isolation in electrical systems [1]. The performance of LFTs (efficiency, volumetric power density, and gravimetric power density) is subject to fundamental physical limitations due to the saturation flux density of the core material, the (thermally) 


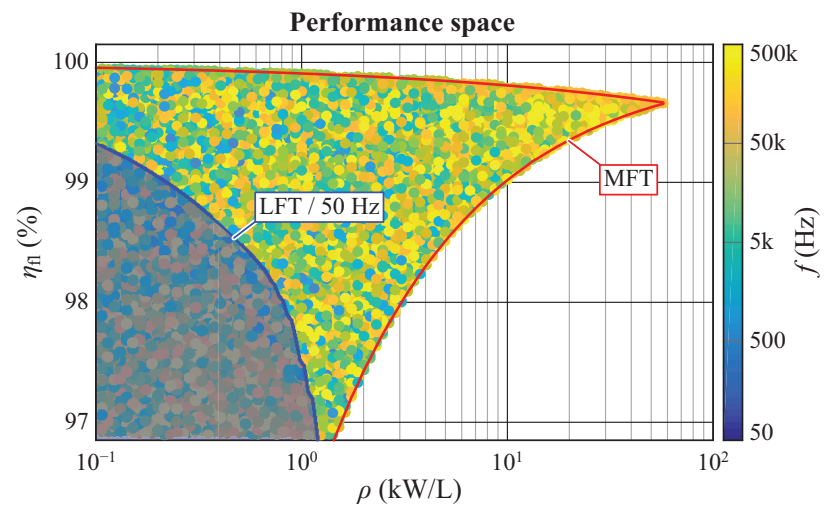

Fig. 1. Comparison between the Pareto fronts of $50 \mathrm{~Hz}$ LFTs and MFTs for a $20 \mathrm{~kW}$ and $600 \mathrm{~V}$ (RMS) system. The LFTs are constructed with solid copper wires and with grain oriented electrical steel cores ("Thyssen Krupp Powercore H 100-27”, 2.0 T saturation flux) [30]. The MFTs are constructed with litz wires (100 $\mu \mathrm{m}$ stranding) and ferrite cores ("TDK N97", $300 \mathrm{mT}$ saturation flux) [31].

limited current density in the windings, and the cooling capabilities [1], [2].

For medium-frequency transformers (MFTs) employed in power electronic converters, the operating frequency is not defined by the grid frequency and, therefore, can be used as an additional degree of freedom, which allows to mitigate (with a reduced volt-second product applied to the magnetic core) the impact of the aforementioned limitations [3]-[9]. Fig. 1 illustrates the Pareto fronts of $50 \mathrm{~Hz}$ LFTs and MFTs for a $20 \mathrm{~kW}$ system. It can be seen that increasing the operating frequency allows for significant improvements in terms of power density and efficiency. It should be noted that, for power electronic converters, medium-frequency is not uniquely defined. In this context, medium-frequency can be quantified with the power-frequency product, which is usually in the range of $[0.05,5.0] \mathrm{MHz}-\mathrm{kW}$, i.e., the absolute operating frequency of a system is dependent on the power level.

However, the usage of medium-frequency introduces new challenges: additional core losses (e.g., due to eddy currents in the core and magnetic relaxation), high frequency losses in the windings (e.g., due to skin and proximity effects), and parasitic resonances [8], [9]. Moreover, MFTs require power electronic converters, which are inverting and rectifying the voltages and currents at the desired operating frequency [4]-[6], [10], [11]. With insulated gate bipolar transistors (IGBTs) (or with hardswitched Silicon MOSFETs), the operating frequency of MFTs is mainly limited by the switching losses of the inverter and rectifier stages. However, with newly available fast-switching Silicon-Carbide (SiC) and Gallium-Nitride $(\mathrm{GaN})$ transistors and/or soft-switching techniques, the switching losses are greatly reduced and the complete design space of MFTs can be used [6], [7], [12]. This implies that a detailed knowledge of the design procedure of MFTs is required for realizing efficient, compact, and inexpensive MFTs utilizing the full potential of modern semiconductors.

Multiple degrees of freedom are available for the design of MFTs: frequency, winding type, winding geometry, core shape, core material, and cooling concept. The analysis of such devices can be split into several categories.

- Equivalent circuit: The MFT is described with an equivalent circuit describing the magnetizing flux, the leakage flux, the magnetic coupling, and the voltage transfer ratio [13], [14].

- Core losses: The core losses (e.g., due to hysteresis and eddy current losses) are usually computed with semi-empirical methods, which are calibrated with measurements [8], [15], [16].

- Winding losses: Different methods exist for computing the winding losses at medium-frequency (e.g., due to skin and proximity effects) for different winding configuration (e.g., litz wire, foil, and printed circuit board (PCB) integrated) [9], [17]-[19].

- Thermal: A thermal model of the MFT and the corresponding cooling system is used for the extraction of the temperature distribution [5], [8], [20].

- Other aspects: Additionally, parasitic capacitances, resonances, electric and/or magnetic shielding, electrical insulation, and dielectric losses should be considered for some designs [7], [8], [21].

The aforementioned aspects are typically combined in order to obtain a full model, which allows for the optimization of MFTs. Such models can be classified into three categories.

- Full-analytical models: The model is based on analytical equations and features a closed-form analytical solution [5], [8], [9], [22]-[24]. The approach is simple and features a clear physical interpretation. Nevertheless, the accuracy of full-analytical models is limited.

- Semi-numerical models: The model is based on analytical equations, but does not feature an explicit solution. The optimal design is obtained with numerical optimization (e.g., brute-force, gradient optimization, and genetic algorithm) [11], [25]-[29]. Such numerical optimizations are accurate and reasonably fast. However, it is difficult to identify the mechanisms leading to the optimal design. Furthermore, the obtained results are specific to the given specifications and cannot be easily generalized.

- Numerical models: The MFT parameters are extracted from numerical field simulations (e.g., finite element method (FEM) simulations) [6], [21]. However, such models are time-consuming (modeling complexity and computational cost) and only provide limited advantages compared to the semi-numerical approach [13]. Therefore, such models are typically not used for optimization processes.

Explicit analytical equations for the design of optimal MFTs have been successfully given in [9], [22], [24], [32]. Analytical scaling laws (scaling of the characteristics of MFTs with respect to a reference design) have been proposed for different power densities, efficiencies, and frequencies in [22], [23], [33], [34]. The complexity of the mapping between the design space and the performance space can be seen in Fig. 1 (mapping of the operating frequency to the performance space) and has also been observed in [12], [32], [35]. However, to the knowledge of the authors, all the aforementioned aspects have not been 


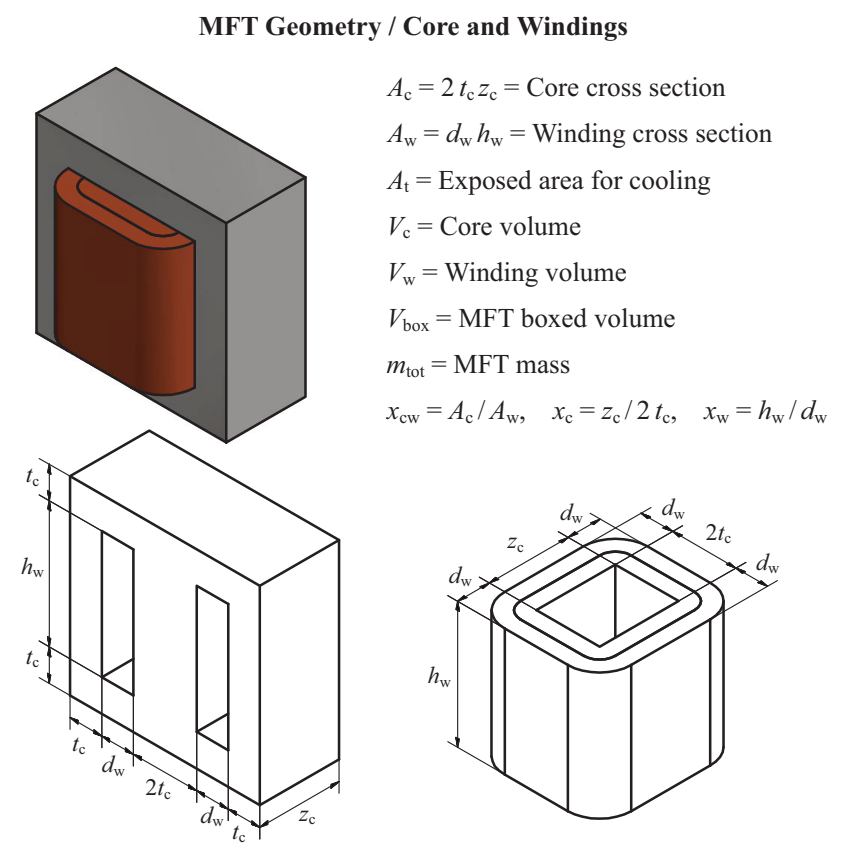

Fig. 2. Considered MFT geometry with E-core, shell-type arrangement, and without winding interleaving. The ratios $x_{\mathrm{cw}}, x_{\mathrm{c}}$, and $x_{\mathrm{w}}$ define the geometrical aspect ratios of the MFT.

brought together up to now. The properties of optimal MFT designs and the scaling of MFTs under different conditions highlight the fundamental characteristics and limitations of MFTs. A better understanding of the mapping between the design space and performance space is also required for using all the available degrees of freedom during multi-objective optimization and for understanding the limitations of the analytical optima.

Therefore, this paper examines the optimization and the scaling of MFTs in detail and is organized as follows. Section II presents the chosen full-analytical MFT model. Section III shows which point in the design space is mapped to the optimal design and which regions are mapped to quasioptimal designs (design space diversity). Moreover, scaling laws are derived for the optimal designs for different power densities, power ratings, temperature rises, and efficiencies. Section IV applies the presented methods to a $20 \mathrm{~kW}$ and $20 \mathrm{~kW} / \mathrm{L}$ $\left(328 \mathrm{~W} /\right.$ in $\left.^{3}\right)$ MFT. Section V successfully compares the results obtained with the full-analytical model with a more elaborate semi-numerical model. Finally, Section VI highlights the limits of the full-analytical models and the corresponding scaling laws.

\section{Full-Analytical Modeling}

In this section, a full-analytical model of MFTs is presented. The core losses, the winding losses, and the thermal limit are considered with simplified analytical models.

\section{A. Geometry and Operating Conditions}

The MFT depicted in Fig. 2 is considered and features the most common design choices: E-core (without air gap) with a shell-type arrangement and litz wire windings (without interleaving). The boxed volume $\left(V_{\text {box }}\right)$ together with the geometrical aspect ratios $\left(x_{\mathrm{cw}}, x_{\mathrm{c}}\right.$, and $\left.x_{\mathrm{w}}\right)$ defines uniquely the geometry of the MFT (cf., Fig. 2). For simplifying the design equations, a turns ratio of $1: 1$ is first selected. The volumetric $(\rho)$ and gravimetric $(\gamma)$ power densities are used as performance indicators for the geometry of the MFT:

$$
\begin{aligned}
& \rho=P / V_{\text {box }}, \\
& \gamma=P / m_{\text {tot }} .
\end{aligned}
$$

In the following, the volumetric power density is primarly considered but all the results can also be obtained for the gravimetric power density. The MFT is assumed to operate with sinusoidal currents and voltages. The apparent power of the MFT $(S)$ can then be expressed with the help of the power factor and the active rated power:

$$
S=P / \cos (\varphi)=I_{\mathrm{RMS}} V_{\mathrm{RMS}} .
$$

The aforementioned design choices have been made since they represent the most typical operation condition of MFTs [4]-[6], [25], [26]. Nevertheless, the presented method can be adapted to other MFT designs and operating conditions, without fundamentally changing the obtained results:

- Transformer type: Transformers with multiple windings (e.g., three-phase transformers) can be considered with small adaptations of the design equations [1], [22].

- Core shape: Other core shapes (e.g., ETD, RM, and U) can be used without any problem by adapting the relations shown in Fig. 2.

- Winding type: Windings composed of solid wires or foils can also be used with minor adaptations of the formulas used for the computations of the losses [5], [8]. Interleaving of the winding can also be considered [8], [9]. Different types of winding arrangements (shell-type or core-type) are also possible [8], [22].

- Turns ratio: The turns ratio of the MFT can be adapted to change the voltage transformation ratio with a small impact on the design variables [5], [22].

- Excitation: Arbitrary (instead of sinusoidal) current and voltage waveforms can be considered with the models presented in [15], [16], [19].

\section{B. Core and Winding Losses}

The core losses of the MFT are computed with the Steinmetz equation, where the lamination factor (if applicable) is included in the Steinmetz parameters [8], [16]. Then, the flux density $\left(B_{\mathrm{pk}}\right)$, the loss density $\left(p_{\mathrm{c}}\right)$, and the core losses $\left(P_{\mathrm{c}}\right)$ can be expressed as

$$
\begin{gathered}
B_{\mathrm{pk}}=\frac{\sqrt{2} V_{\mathrm{RMS}}}{2 \pi n f A_{\mathrm{c}}}, \\
p_{\mathrm{c}}=k f^{\alpha_{\mathrm{c}}} B_{\mathrm{pk}}^{\beta_{\mathrm{c}}}, \\
P_{\mathrm{c}}=V_{\mathrm{c}} p_{\mathrm{c}},
\end{gathered}
$$


This model neglects the frequency and temperature dependences of the Steinmetz parameters [15], [20]. In addition, the following limitations (saturation flux and maximal operating frequency of the core material) apply to the aforementioned model:

$$
\begin{gathered}
n>\frac{\sqrt{2} V_{\mathrm{RMS}}}{2 \pi f B_{\mathrm{sat}} A_{\mathrm{c}}} \Leftrightarrow B_{\mathrm{pk}}<B_{\mathrm{sat}}, \\
f<f_{c, \text { max }} .
\end{gathered}
$$

The Steinmetz equation is an empirical model which is parametrized with measurement data. The accuracy of the model (in the relevant frequency and flux density ranges) should be checked for every considered core material.

The winding losses of litz wire windings (shell-type arrangement) are computed with the asymptotic approximation of the proximity effect losses (without interleaving) [5], [19], [22], [24]. Then, the current density $\left(J_{\mathrm{RMS}}\right)$, the proximity effect factor $\left(a_{\mathrm{w}}\right)$, the skin depth $(\delta)$, the loss density $\left(p_{\mathrm{w}}\right)$, and the winding losses $\left(P_{\mathrm{w}}\right)$ can be expressed as

$$
\begin{aligned}
J_{\mathrm{RMS}} & =\frac{2 n I_{\mathrm{RMS}}}{k_{\mathrm{w}} A_{\mathrm{w}}}, \\
a_{\mathrm{w}} & =\frac{1}{24}\left(\pi \mu_{0} \sigma k_{\mathrm{w}} d_{\mathrm{w}} d_{\mathrm{S}}\right)^{2}, \\
\delta & =\frac{1}{\sqrt{\pi \sigma \mu_{0} f}}, \\
p_{\mathrm{w}} & =\left(1+a_{\mathrm{w}} f^{2}\right) \frac{J_{\mathrm{RMS}}^{2}}{\sigma}, \\
P_{\mathrm{w}} & =\left(V_{\mathrm{w}} k_{\mathrm{w}}\right) p_{\mathrm{w}} .
\end{aligned}
$$

This model neglects the temperature dependence of the winding losses, the exact placement of the wires, and the additional losses occurring when the skin depth reaches the strand diameter [13], [18]. The filling factor considers the packing of the strands (litz wire), the packing of the turns, and the insulation distances. The following limitations (maximal current density and skin depth) apply to the aforementioned model:

$$
\begin{gathered}
n<\frac{k_{\mathrm{w}} A_{\mathrm{w}} J_{\mathrm{RMS}, \max }}{2 I_{\mathrm{RMS}}} \Leftrightarrow J_{\mathrm{RMS}}<J_{\mathrm{RMS}, \max }, \\
f<\frac{1}{\pi \sigma \mu_{0} d_{\mathrm{s}}^{2}} \Leftrightarrow d_{\mathrm{s}}<\delta .
\end{gathered}
$$

Within the aforementioned limits, this model represents a good approximation of the high-frequency winding losses (less than $10 \%$ error compared to FEM simulations) as analyzed in [13], [22].

From the core and winding losses, different performance indicators can be extracted. The ratio between the core and winding losses $\left(r_{\mathrm{cw}}\right)$, the ratio between the $\mathrm{AC}$ and $\mathrm{DC}$ winding resistances $\left(r_{\mathrm{w}}\right)$, the total MFT losses $\left(P_{1}\right)$, and the efficiencies $\left(\eta_{\mathrm{fl}}\right.$ and $\left.\eta_{\mathrm{pl}}\right)$ can be expressed as

$$
\begin{aligned}
r_{\mathrm{cw}} & =\frac{P_{\mathrm{c}}}{P_{\mathrm{w}}}, \\
r_{\mathrm{w}} & =1+a_{\mathrm{w}} f^{2}, \\
P_{1} & =P_{\mathrm{w}}+P_{\mathrm{c}}, \\
\eta_{\mathrm{fl}} & =1-\frac{\left(P_{\mathrm{w}}+P_{\mathrm{c}}\right)}{P}, \\
\eta_{\mathrm{pl}} & =1-\frac{\left(P_{\mathrm{w}} / 4+P_{\mathrm{c}}\right)}{P / 2} .
\end{aligned}
$$

\section{Thermal Modeling}

A thermal model is required for ensuring that the MFT is not operated beyond the thermal limit. However, complex thermal models [5], [15], [26], [29], [36] are not adapted for deriving scaling laws. A model based on the convection coefficient and the exposed area of the MFT [23], [24] has been selected. The convection coefficient $\left(h_{\mathrm{t}}\right)$ is obtained with a fit (temperature and exposed area dependences) of the empirical formulas provided in [8]:

$$
h_{\mathrm{t}}=k_{\mathrm{t}} \Delta T^{v_{\mathrm{t}}} A_{\mathrm{t}}^{\kappa_{\mathrm{t}}} \text {. }
$$

This model can be used to describe natural and/or forced convection processes. Heat conduction and radiation processes are neglected. Then, the temperature rise $(\Delta T)$ of the transformer (windings and core) and the corresponding thermal limit can be expressed as

$$
\begin{aligned}
\Delta T=\frac{P_{1}}{h_{\mathrm{t}} A_{\mathrm{t}}} & =\left(\frac{P_{1}}{k_{\mathrm{t}} A_{\mathrm{t}}^{1+\kappa_{\mathrm{t}}}}\right)^{\frac{1}{1+v_{\mathrm{t}}}} \\
\Delta T & <\Delta T_{\text {max }} .
\end{aligned}
$$

\section{Optimization and Scaling LaWs}

In this section, the presented full-analytical model (cf., Section II) is used to derive conditions for optimal designs. Furthermore, the properties of the optimal designs are analyzed for different frequencies, power densities, and power ratings.

\section{A. Area Product}

The area product (product of the core and winding window cross sections) is a common method for designing LFTs [8], [23]. The area product, which is directly linked to the LFT power rating, can be expressed as

$$
A_{\mathrm{c}} A_{\mathrm{w}}=\frac{\sqrt{2} V_{\mathrm{RMS}}}{2 \pi f n B_{\mathrm{pk}}} \frac{2 n I_{\mathrm{RMS}}}{k_{\mathrm{w}} J_{\mathrm{RMS}}}=\frac{\sqrt{2}}{\pi k_{\mathrm{w}} B_{\mathrm{pk}} J_{\mathrm{RMS}}} \frac{S}{f} .
$$




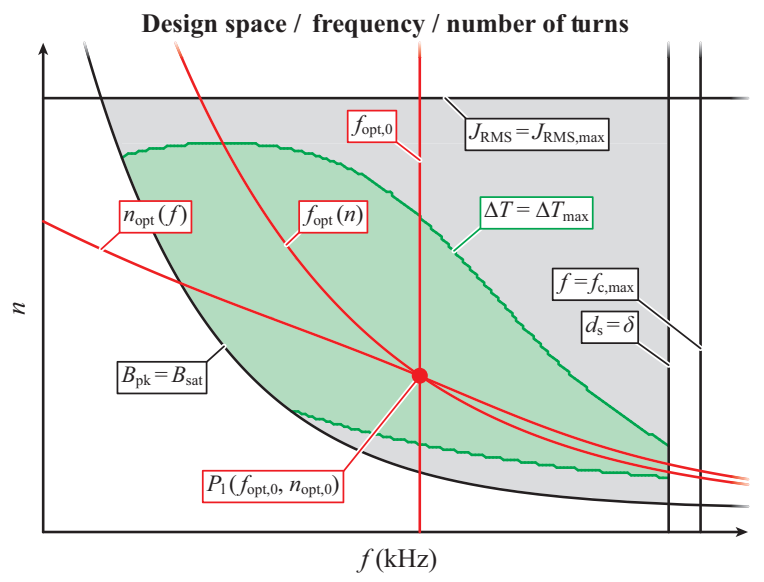

Fig. 3. MFT design space for different frequencies and numbers of turns, assuming a constant area product and/or core size. The design space limits (black and gray), the thermal limit (green), and the optimal designs (red) are shown.

For LFTs, the core losses are low and the peak flux density $\left(B_{\mathrm{pk}}\right)$ can be set to the saturation flux density level $\left(B_{\mathrm{sat}}\right)$ and the current density $\left(J_{\mathrm{RMS}}\right)$ to the maximum value $\left(J_{\mathrm{RMS}, \max }\right)$. Then, the area product represents a valuable design equation for obtaining a first approximation of a LFT size and performance. The area product also indicates that the operation at an increased frequency will lead to more compact designs.

However, for MFTs, the optimal flux and current densities can be significantly lower than the maximum values [5], [24], [33]. This is due to the increased core losses (parameter $\alpha_{\mathrm{c}}$ ) and the proximity effect losses in the windings (parameter $a_{\mathrm{w}}$ ). This implies that the area product cannot be directly used for designing MFTs. Nevertheless, the area product still represents an upper bound for MFTs, which can be useful as a starting point during the design process.

\section{B. Optimal MFT Design}

With the aforementioned models (cf., Section II), it is possible to derive a closed-form solution for optimal MFTs [5], [22]. However, several assumptions are required for obtaining a simple solution:

- The power rating, power factor, and voltage level $(P, \cos (\varphi)$, and $\left.V_{\text {RMS }}\right)$ are fixed. Therefore, it is accepted that the reactive power is not strongly dependent on the leakage and magnetizing inductances of the different designs. This is the case for MFTs with high magnetic coupling factors [13].

- The geometry is fixed $\left(V_{\mathrm{box}}, x_{\mathrm{cw}}, x_{\mathrm{c}}\right.$, and $\left.x_{\mathrm{w}}\right)$. This corresponds to a specific magnetic core size.

- The core material $\left(B_{\text {sat }}, k_{\mathrm{c}}, \alpha_{\mathrm{c}}\right.$, and $\left.\beta_{\mathrm{c}}\right)$ and the winding stranding $\left(J_{\mathrm{RMS}, \max }, k_{\mathrm{w}}, \sigma\right.$, and $\left.d_{\mathrm{s}}\right)$ are selected, i.e., not variable.

- The number of turns $(n)$ is accepted to be a continuous (non-discrete) variable. The winding filling factor is independent of the number of turns and the exact packing pattern of the turns is not considered.
With these assumptions, the only remaining degrees of freedom are the operating frequency $(f)$ and the number of turns $(n)$. This design space is constrained by the saturation flux density (cf., (7)), the current density (cf., (14)), and the maximum frequency (cf., (8) and (15)). Fig. 3 shows the resulting design space. With the presented models, the MFT losses can be expressed as

$$
\begin{gathered}
P_{\mathrm{c}}(f, n)=C_{\mathrm{o}} f^{\alpha_{\mathrm{c}}-\beta_{\mathrm{c}}-\beta_{\mathrm{c}}}, \\
P_{\mathrm{w}}(f, n)=C_{\mathrm{w}}\left(1+a_{\mathrm{w}} f^{2}\right) n^{2}, \\
P_{1}(f, n)=P_{\mathrm{c}}(f, n)+P_{\mathrm{w}}(f, n),
\end{gathered}
$$

where the following constants are defined:

$$
\begin{gathered}
C_{\mathrm{c}}=\left(k_{\mathrm{c}} V_{\mathrm{c}}\right)\left(\frac{\sqrt{2} V_{\mathrm{RMS}}}{2 \pi A_{\mathrm{c}}}\right)^{\beta_{\mathrm{c}}}, \\
C_{\mathrm{w}}=\frac{4 V_{\mathrm{w}} I_{\mathrm{RMS}}^{2}}{\sigma k_{\mathrm{w}} A_{\mathrm{w}}^{2}} .
\end{gathered}
$$

Now, the optimal number of turns for a given frequency $\left(n_{\mathrm{opt}}\right)$ and the optimal frequency $\left(f_{\mathrm{opt}}\right)$ for a given number of turns can be calculated (cf., Fig. 3). The following expressions can be calculated with partial derivatives:

$$
\begin{aligned}
& \frac{\partial P_{1}(f, n)}{\partial n}=0 \Rightarrow n_{\mathrm{opt}}(f)=\left(\frac{C_{\mathrm{c}} \beta_{\mathrm{c}} f^{\alpha_{\mathrm{c}}-\beta_{\mathrm{c}}}}{2 C_{\mathrm{w}}\left(1+a_{\mathrm{w}} f^{2}\right)}\right)^{\frac{1}{2+\beta_{\mathrm{c}}}}, \\
& \frac{\partial P_{1}(f, n)}{\partial f}=0 \Rightarrow f_{\mathrm{opt}}(n)=\left(\frac{2 C_{\mathrm{w}} a_{\mathrm{w}} n^{2+\beta_{\mathrm{c}}}}{C_{\mathrm{c}}\left(\beta_{\mathrm{c}}-\alpha_{\mathrm{c}}\right)}\right)^{\frac{1}{\alpha_{\mathrm{c}}-\beta_{\mathrm{c}}-2}} .
\end{aligned}
$$

It should be noted that the optimal frequency $\left(f_{\text {opt }}\right)$ only exists if $\beta_{\mathrm{c}}>\alpha_{\mathrm{c}}$, which is almost always respected for core materials operated at typical frequencies. For $\beta_{\mathrm{c}}=\alpha_{\mathrm{c}}$, the optimal frequency would be zero, and all the designs would be operated at the saturation limit [33]. The optimal number of turns $\left(n_{\mathrm{opt}}\right)$ can be very low or very high, which can lead to practical problems for the construction of the windings. However, the number of turns can be adapted by changing the core geometrical aspect ratios, i.e., the ratio between the core $\left(A_{\mathrm{c}}\right)$ and windings $\left(A_{\mathrm{w}}\right)$ areas (cf., Fig. 2).

The intersection between $n_{\text {opt }}$ and $f_{\text {opt }}$ represents the global optimum (gradient of the loss function is zero, cf., Fig. 3) and can be expressed as

$$
f_{\mathrm{opt}, 0}=\sqrt{\frac{\beta_{\mathrm{c}}-\alpha_{\mathrm{c}}}{\alpha_{\mathrm{c}} a_{\mathrm{w}}}}, \quad n_{\mathrm{opt}, 0}=n_{\mathrm{opt}}\left(f_{\mathrm{opt}, 0}\right) .
$$

This implies that beyond $f_{\mathrm{opt}, 0}$, the increase of the proximity effect losses in the windings exceeds the reduction of the core losses. The designs with the optimal number of turns feature 
the following property between the core and winding losses (at any frequency as long as the saturation flux, maximum current density, and maximum frequency are not reached) [5], [22], [24]:

$$
n=n_{\mathrm{opt}}(f) \Rightarrow r_{\mathrm{cw}}=\frac{2}{\beta_{\mathrm{c}}} \text {. }
$$

The global optimum, additionally, features a specific ratio between the AC and DC winding resistances [5], [22], [24]:

$$
\left(f=f_{\mathrm{op}, 0}\right) \wedge\left(n=n_{\mathrm{opt}, 0}\right) \Rightarrow\left(r_{\mathrm{w}}=\frac{\beta_{\mathrm{c}}}{\alpha_{\mathrm{c}}}\right) \wedge\left(r_{\mathrm{cw}}=\frac{2}{\beta_{\mathrm{c}}}\right) .
$$

The global optimum can be situated outside the design space, especially for designs with large power densities. Then, the feasible optimum will be located at the boundary of the design space, typically at the core saturation limit. Furthermore, the thermal limit is also restricting the valid designs around the global optimum (cf., Fig. 3). However, MFTs are typically operated below the saturation, frequency, and current density limits [4]-[6], [11] and, therefore, the derived optimum can be used. Hence, the nature of this global optimum is analyzed in more details in the next subsection.

\section{Frequency Diversity}

In the design space shown in Fig. 3, all the designs with an optimal number of turns $\left(n_{\mathrm{opt}}\right.$, as long the saturation flux is not reached) are considered for different frequencies. This restriction is accepted since there are no reasons to build a MFT with a non-optimal number of turns. By contrast, due to the switching losses of the power semiconductors and/or electro magnetic interference (EMI) issues, it can be desirable to select an operating frequency below $f_{\mathrm{opt}, 0}$. The impact of the selection of a sub-optimal operating frequency can be described with the following equation:

$$
\varepsilon=\max \left[\frac{P_{1}\left(f, n_{\mathrm{opt}}\right)}{P_{1}\left(f_{\mathrm{opt}, 0}, n_{\mathrm{opt}, 0}\right)}-1\right] \text {, with } \frac{f_{\mathrm{opt}, 0}}{f}<\xi,
$$

where $\xi$ represents the allowed relative deviation compared to the optimal frequency $\left(f_{\text {opt }, 0}\right)$ and $\varepsilon$ the corresponding relative impact on the losses, as illustrated in Fig. 4(a).

The resulting characteristic of the losses curve is rather flat with respect to frequency $\left(f<f_{\text {opt }, 0}\right)$ as long as the saturation flux is not reached ( $n_{\text {opt }}$ is located inside the design space). As soon as $n_{\text {opt }}$ crosses the saturation limit, the number of turns should be selected with respect to the saturation limit (and not anymore with $n_{\mathrm{opt}}$ ). This leads to a rapid increase of the winding losses and the optimal ratios between the core and winding losses (cf., (33)) cannot be anymore achieved. Therefore, at the saturation limit, the total losses are rapidly increasing.

The aforementioned equation can be solved analytically if all the designs are located inside the design space limitations

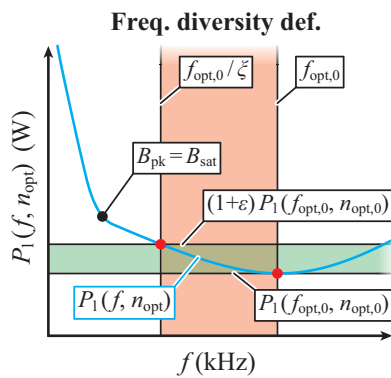

(a)

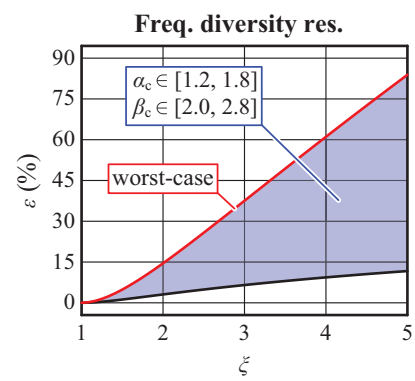

(b)
Fig. 4. (a) Definition of the frequency diversity (cf., (35)) with reference to the global optimum (cf., (34)). For the different frequencies $\left(f \neq f_{\text {opt }, 0}\right)$, the respective optimal number of turns $\left(n_{\mathrm{opt}}\right)$ is considered, as long the saturation flux is not reached $\left(B_{\mathrm{pk}}<B_{\mathrm{sat}}\right)$. (b) Obtained frequency diversity (cf., (36)) considering a typical range of Steinmetz parameters.

( $n_{\text {opt }}$ is not limited by the saturation flux), which is the case for typical MFT designs. This leads to

$$
\varepsilon=\left(\frac{1}{\xi^{2}}\right)^{\frac{\alpha_{c}}{2+\beta_{c}}}\left(\frac{\beta_{c}-\alpha_{c}\left(1-\xi^{2}\right)}{\beta_{c}}\right)^{\frac{\beta_{c}}{2+\beta_{c}}}-1,
$$

where it should be noted that the solution of the equation is only dependent on $\alpha_{\mathrm{c}}, \beta_{\mathrm{c}}$, and $\xi$. Therefore, the solution can be evaluated numerically for a range of Steinmetz parameters covering typical core materials [8], [31], [37]-[42].

Fig. 4(b) shows the obtained results. It can be observed that frequencies significantly below $f_{\mathrm{opt}, 0}$ can be selected with a minor impact on the efficiency. For example, with half of the optimal frequency $(\xi=2)$, the losses are, at most, only $15 \%$ higher than the global optimum. It can be concluded that the MFT loss curve is always flat around $f_{\mathrm{opt}, 0}$. This implies that the optimum $\left(f_{\text {opt }, 0}\right)$ should be considered carefully for converter designs. A small amount of frequency-dependent losses in the converter (e.g., switching losses of the semiconductors) can significantly shift down the optimal operating frequency of the complete converter with respect to the MFT optimum, however, with a minor impact on the achieved performances.

Nevertheless, if the selected operating frequency is significantly below $f_{\text {opt }, 0}$, this indicates that a less expensive core material and litz wire could be selected with a minor impact on the achieved performance. However, the optimal frequency $\left(f_{\mathrm{opt}, 0}\right)$ is interesting for examining the fundamental design limitations of power converters. This holds particularly true with modern $\mathrm{SiC}$ and $\mathrm{GaN}$ transistors, where the magnetic components often limit the achievable efficiency and power density [12]. Therefore, scaling laws are derived for the optimal MFT $\left(f_{\mathrm{opt}, 0}\right.$ and $\left.n_{\mathrm{opt}, 0}\right)$ in the next subsection.

\section{Scaling Laws}

The scaling of MFTs is considered for different power ratings $(P)$ and power densities $(\rho)$ [23], [34]. Different hypotheses are made in order to obtain simple and general scaling laws:

- The power factor and voltage levels $\left(\cos (\varphi)\right.$, and $\left.V_{\mathrm{RMS}}\right)$ are fixed. The power rating is scaled with the current. However, 


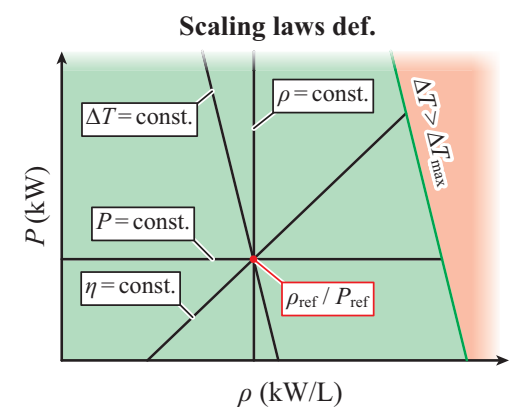

(a)
Scaling laws var.

\begin{tabular}{c}
\hline Variable ranges: \\
$\alpha_{\mathrm{c}} \in[+1.2,+1.8]$ \\
$\beta_{\mathrm{c}} \in[+2.0,+2.8]$ \\
$v_{\mathrm{t}} \in[+0.0,+0.2]$ \\
$\kappa_{\mathrm{t}} \in[-0.2,+0.0]$ \\
\hline Assumptions: \\
$f=f_{\mathrm{opt}, 0} / n=n_{\mathrm{opt}, 0}$ \\
\hline
\end{tabular}

(b)
Fig. 5. (a) Scaling of MFTs for different power ratings $(P)$ and power densities $(\rho)$ with characteristics for constant power rating, constant power density, constant temperature rise, and constant efficiency. For the scaling, the global optimum (cf., (34)) is considered with a given set of (constant) parameters for the core material $\left(\alpha_{\mathrm{c}}\right.$ and $\left.\beta_{\mathrm{c}}\right)$ and the heat transfer mechanism $\left(v_{\mathrm{t}}\right.$ and $\left.\kappa_{\mathrm{t}}\right)$. (b) Considered parameter ranges and assumptions for Tables I, II, III, and IV.

the impedance of the MFT (ratio between $V_{\mathrm{RMS}}$ and $I_{\mathrm{RMS}}$ ) has only a minor impact on the derived scaling laws.

- The volume (or power density) of the MFT is scaled with fixed (constant) geometrical aspect ratios $\left(x_{\mathrm{cw}}, x_{\mathrm{c}}\right.$, and $\left.x_{\mathrm{w}}\right)$.

- The core material $\left(B_{\mathrm{sat}}, k_{\mathrm{c}}, \alpha_{\mathrm{c}}\right.$, and $\left.\beta_{\mathrm{c}}\right)$ and the winding stranding $\left(J_{\mathrm{RMS}, \max }, k_{\mathrm{w}}, \sigma\right.$, and $\left.d_{\mathrm{s}}\right)$ are fixed.

- The number of turns $(n)$ is accepted to be a continuous (non-discrete) variable. The winding filling factor is independent of the number of turns, the power rating, and the power density.

- The global optimum is considered for all the designs $\left(f_{\text {opt }, 0}\right.$ and $\left.n_{\mathrm{opt}, 0}\right)$. It is accepted that the global optimum is located inside (and not at the boundary) of the design space (cf., Fig. 3), which is the case for typical MFT designs.

With the aforementioned hypotheses, it is possible to derive analytical scaling laws for the power density $(\rho)$ and the power rating $(P)$ :

$$
\begin{aligned}
& \frac{x}{x_{\text {ref }}}=\left(\frac{\rho}{\rho_{\text {ref }}}\right)^{\lambda} \mid\left(f=f_{\mathrm{opt}, 0}\right) \wedge\left(n=n_{\mathrm{op}, 0}\right), \\
& \frac{x}{x_{\text {ref }}}=\left(\frac{P}{P_{\text {ref }}}\right)^{\lambda} \mid\left(f=f_{\mathrm{op}, 0}\right) \wedge\left(n=n_{\mathrm{op}, 0}\right),
\end{aligned}
$$

where $\rho_{\text {ref }}$ and $P_{\text {ref }}$ represents the reference design and $\lambda$ the scaling exponent. Each characteristic, $x$, can be scaled with respect to the reference value, $x_{\text {ref }}$. The following characteristics are considered: the power rating $(P)$, the volumetric power density $(\rho)$, the gravimetric power density $(\gamma)$, the temperature rise $(\Delta T)$, the loss fraction $\left(1-\eta_{\mathrm{fl}}\right.$ and $\left.1-\eta_{\mathrm{pl}}\right)$, the frequency $\left(f_{\mathrm{op}, 0}\right)$, the number of turns $\left(n_{\mathrm{opt}, 0}\right)$, the peak flux density $\left(B_{\mathrm{pk}}\right)$, the current density $\left(J_{\mathrm{RMS}}\right)$, the $\mathrm{AC} / \mathrm{DC}$ winding resistance ratio $\left(r_{\mathrm{w}}\right)$, and the ratio between the core and winding losses $\left(r_{\mathrm{cw}}\right)$. It should be noted that simple exponential scaling laws only exist due to the special properties of the global optimum (cf., (34)).

There are several ways of scaling a MFT for different power ratings and power densities. However, in order to give meaningful results, certain parameters and/or performance indicators should be kept constant, as shown in Fig. 5(a) [23]:
TABLE I

SCAling CoefF. $/ P=$ const. $/ \rho=$ var.

\begin{tabular}{lcc}
\hline \hline$x$ & Coeff. $(\lambda)$ & Range $(\lambda)$ \\
\hline$P$ & 0 & {$[+0.00,+0.00]$} \\
$\rho$ & 1 & {$[+1.00,+1.00]$} \\
\hline$\gamma$ & 1 & {$[+1.00,+1.00]$} \\
$\Delta T$ & $\frac{2 \alpha_{\mathrm{c}}+5 \beta_{\mathrm{c}}+4 \kappa_{\mathrm{t}}+2 \beta_{\mathrm{c}} \kappa_{\mathrm{t}}-2}{3\left(\beta_{\mathrm{c}}+2\right)\left(v_{\mathrm{t}}+1\right)}$ & {$[+0.61,+1.08]$} \\
$1-\eta_{\mathrm{pl}}$ & $\frac{2 \alpha_{\mathrm{c}}+3 \beta_{\mathrm{c}}-6}{3 \beta_{\mathrm{c}}+6}$ & {$[+0.20,+0.42]$} \\
$1-\eta_{\mathrm{fl}}$ & $\frac{2 \alpha_{\mathrm{c}}+3 \beta_{\mathrm{c}}-6}{3 \beta_{\mathrm{c}}+6}$ & {$[+0.20,+0.42]$} \\
\hline$f_{\mathrm{opt}, 0}$ & $\frac{1}{3}$ & {$[+0.33,+0.33]$} \\
$n_{\mathrm{opt}, 0}$ & $\frac{\alpha_{\mathrm{c}}+\beta_{\mathrm{c}}-4}{3 \beta_{\mathrm{c}}+6}$ & {$[-0.07,+0.04]$} \\
\hline$B_{\mathrm{pk}}$ & $\frac{6-\alpha_{\mathrm{c}}}{3 \beta_{\mathrm{c}}+6}$ & {$[+0.29,+0.40]$} \\
$J_{\mathrm{RMS}}$ & $\frac{\alpha_{\mathrm{c}}+3 \beta_{\mathrm{c}}}{3 \beta_{\mathrm{c}}+6}$ & {$[+0.60,+0.71]$} \\
$r_{\mathrm{W}}$ & 0 & {$[+0.00,+0.00]$} \\
$r_{\mathrm{cW}}$ & 0 & {$[+0.00,+0.00]$} \\
\hline \hline
\end{tabular}

- $P=$ const. - The power rating of the MFT is constant and the power density is scaled (cf., (37)).

- $\rho=$ const. - The power density of the MFT is constant and the power rating is scaled (cf., (38)).

- $\eta=$ const. - The power rating of the MFT is scaled with a constant efficiency (cf., (38)).

- $\Delta T=$ const. - The power rating of the MFT is scaled with a constant temperature rise (cf., (38)).

Exact analytical solutions exist for the scaling coefficients $(\lambda)$. The solutions only depend on the empirical parameters $\alpha_{\mathrm{c}}, \beta_{\mathrm{c}}, v_{\mathrm{t}}$, and $\kappa_{\mathrm{t}}$. For extracting general statements, the scaling coefficients are extracted for all the parameter combinations occurring for typical core materials and convective heat transfer mechanisms, cf., Fig. 5(b) [8], [31], [37]-[42]. The power rating of the MFT is scaled with a constant voltage. However, it should be noted that all the parameters, except the number of turns $\left(n_{\mathrm{opt}, 0}\right)$, are independent of the impedance of the MFT (i.e., the ratio between $V_{\mathrm{RMS}}$ and $I_{\mathrm{RMS}}$ ).

Table I shows the scaling coefficients for a constant power rating $(P=$ const.). For increasing power densities, the frequency, and temperature rise are increasing, until the thermal limit is reached. The loss fraction is slowly increasing with the power density, indicating that the Pareto front is flat with respect to the power density.

Table II depicts the scaling coefficients for a constant power density ( $\rho=$ const.). For increasing power ratings, the temperature rise is increasing while the frequency is decreasing. The loss fraction is also slowly decreasing with the power rating, implying that high power MFTs are intrinsically more efficient but difficult to cool down.

Table III shows the scaling coefficients for a constant efficiency ( $\eta=$ const.). For increasing power ratings, the power density is increasing while the frequency is decreasing. The temperature rise is also increasing, which implies that high power designs are hitting the thermal limit. This is due to the reduction of the ratio between the surface area $\left(A_{\mathrm{h}}\right)$ and the 
TABLE II

ScAling Coeff. $/ \rho=$ const. $/ P=$ var.

\begin{tabular}{lcc}
\hline \hline$x$ & Coeff. $(\lambda)$ & Range $(\lambda)$ \\
\hline$\rho$ & 0 & {$[+0.00,+0.00]$} \\
$P$ & 1 & {$[+1.00,+1.00]$} \\
\hline$\gamma$ & 0 & {$[+0.00,+0.00]$} \\
$\Delta T$ & $\frac{\beta_{\mathrm{c}}-2 \alpha_{\mathrm{c}}-4 \kappa_{\mathrm{t}}-2 \beta_{\mathrm{c}} \kappa_{\mathrm{t}}+2}{3\left(\beta_{\mathrm{c}}+2\right)\left(v_{\mathrm{t}}+1\right)}$ & {$[+0.03,+0.30]$} \\
$1-\eta_{\mathrm{pl}}$ & $\frac{-2 \alpha_{\mathrm{c}}}{3 \beta_{\mathrm{c}}+6}$ & {$[-0.30,-0.17]$} \\
$1-\eta_{\mathrm{fl}}$ & $\frac{-2 \alpha_{\mathrm{c}}}{3 \beta_{\mathrm{c}}+6}$ & {$[-0.30,-0.17]$} \\
\hline$f_{\mathrm{opt}, 0}$ & $-\frac{1}{3}$ & {$[-0.33,-0.33]$} \\
$n_{\mathrm{opt}, 0}$ & $\frac{-\alpha_{\mathrm{c}}-\beta_{\mathrm{c}}-2}{3 \beta_{\mathrm{c}}+6}$ & {$[-0.48,-0.42]$} \\
\hline$B_{\mathrm{pk}}$ & $\frac{\alpha_{\mathrm{c}}}{3 \beta_{\mathrm{c}}+6}$ & {$[+0.08,+0.15]$} \\
$J_{\mathrm{RMS}}$ & $\frac{-\alpha_{\mathrm{c}}}{3 \beta_{\mathrm{c}}+6}$ & {$[-0.15,-0.08]$} \\
$r_{\mathrm{W}}$ & 0 & {$[+0.00,+0.00]$} \\
$r_{\mathrm{cW}}$ & 0 & {$[+0.00,+0.00]$} \\
\hline \hline
\end{tabular}

TABLE III

SCALING COEFF. $/ \eta=$ const. $/ P=$ var

\begin{tabular}{lcc}
\hline \hline$x$ & Coeff. $(\lambda)$ & Range $(\lambda)$ \\
\hline $1-\eta_{\mathrm{pl}}$ & 0 & {$[+0.00,+0.00]$} \\
$1-\eta_{\mathrm{fl}}$ & 0 & {$[+0.00,+0.00]$} \\
$P$ & 1 & {$[+1.00,+1.00]$} \\
\hline$\rho$ & $\frac{2 \alpha_{\mathrm{c}}}{2 \alpha_{\mathrm{c}}+3 \beta_{\mathrm{c}}-6}$ & {$[+0.50,+1.00]$} \\
$\gamma$ & $\frac{2 \alpha_{\mathrm{c}}}{2 \alpha_{\mathrm{c}}+3 \beta_{\mathrm{c}}-6}$ & {$[+0.50,+1.00]$} \\
$\Delta T$ & $\frac{2 \alpha_{\mathrm{c}}+\beta_{\mathrm{c}}+4 \kappa_{\mathrm{t}}-2 \beta_{\mathrm{c}} \kappa_{\mathrm{t}}-2}{\left(v_{\mathrm{t}}+1\right)\left(2 \alpha_{\mathrm{c}}+3 \beta_{\mathrm{c}}-6\right)}$ & {$[+0.56,+1.00]$} \\
\hline$f_{\mathrm{opt}, 0}$ & $\frac{2-\beta_{\mathrm{c}}}{2 \alpha_{\mathrm{c}}+3 \beta_{\mathrm{c}}-6}$ & {$[-0.17,-0.00]$} \\
$n_{\mathrm{opt}, 0}$ & $\frac{2-\beta_{\mathrm{c}}-\alpha_{\mathrm{c}}}{2 \alpha_{\mathrm{c}}+3 \beta_{\mathrm{c}}-6}$ & {$[-0.50,-0.42]$} \\
\hline$B_{\mathrm{pk}}$ & $\frac{\alpha_{\mathrm{c}}}{2 \alpha_{\mathrm{c}}+3 \beta_{\mathrm{c}}-6}$ & {$[+0.25,+0.50]$} \\
$J_{\mathrm{RMS}}$ & $\frac{\alpha_{\mathrm{c}}}{2 \alpha_{\mathrm{c}}+3 \beta_{\mathrm{c}}-6}$ & {$[+0.25,+0.50]$} \\
$r_{\mathrm{W}}$ & 0 & {$[+0.00,+0.00]$} \\
$r_{\mathrm{cW}}$ & 0 & {$[+0.00,+0.00]$} \\
\hline \hline
\end{tabular}

volume $\left(V_{\text {box }}\right)$ for large MFTs.

Table IV shows the scaling coefficients for a constant temperature rise ( $\Delta T=$ const.). For increasing power ratings, the power density and the frequency are decreasing. The loss fraction is decreasing, implying that high power MFTs should be particularly efficient for staying thermally feasible. The same trend can also be observed for LFTs [1], [2].

General trends can be identified independent of the chosen core material and convective cooling mechanisms (cf., Fig. 5(b)). The following conclusions are drawn from the scaling laws. Compact designs are running with higher frequencies, temperature rises, flux densities, and current densities but feature limited efficiencies. High power designs feature lower frequencies and higher efficiencies but limited cooling capabilities.

Scaling laws can also be derived for a fixed frequency (different
TABLE IV

SCALING CoEfF. $/ \Delta T=$ const. $/ P=$ var.

\begin{tabular}{lcc}
\hline \hline$x$ & Coeff. $(\lambda)$ & Range $(\lambda)$ \\
\hline$\Delta T$ & 0 & {$[+0.00,+0.00]$} \\
$P$ & 1 & {$[+1.00,+1.00]$} \\
\hline$\rho$ & $\frac{2 \alpha_{\mathrm{c}}-\beta_{\mathrm{c}}+4 \kappa_{\mathrm{t}}+2 \beta_{\mathrm{c}} \kappa_{\mathrm{t}}-2}{22 \alpha_{\mathrm{c}}+5 \beta_{\mathrm{c}}+4 \kappa_{\mathrm{t}}+2 \beta_{\mathrm{c}} \kappa_{\mathrm{t}}-2}$ & {$[-0.36,-0.03]$} \\
$\gamma$ & $\frac{2 \alpha_{\mathrm{c}}-\beta_{\mathrm{c}}+4 \kappa_{\mathrm{t}}+2 \beta_{\mathrm{c}} \kappa_{\mathrm{t}}-2}{2 \alpha_{\mathrm{c}}+5 \beta_{\mathrm{c}}+4 \kappa_{\mathrm{t}}+2 \beta_{\mathrm{c}} \kappa_{\mathrm{t}}-2}$ & {$[-0.36,-0.03]$} \\
$1-\eta_{\mathrm{pl}}$ & $\frac{2 \beta_{\mathrm{c}} \kappa_{\mathrm{t}}-\beta_{\mathrm{c}}-4 \kappa_{\mathrm{t}}-2 \alpha_{\mathrm{c}}+2}{2 \alpha_{\mathrm{c}}+5 \beta_{\mathrm{c}}+4 \kappa_{\mathrm{t}}+2 \beta_{\mathrm{c}} \kappa_{\mathrm{t}}-2}$ & {$[-0.36,-0.22]$} \\
$1-\eta_{\mathrm{fl}}$ & $\frac{2 \beta_{\mathrm{c}} \kappa_{\mathrm{t}}-\beta_{\mathrm{c}}-4 \kappa_{\mathrm{t}}-2 \alpha_{\mathrm{c}}+2}{2 \alpha_{\mathrm{c}}+5 \beta_{\mathrm{c}}+4 \kappa_{\mathrm{t}}+2 \beta_{\mathrm{c}} \kappa_{\mathrm{t}}-2}$ & {$[-0.36,-0.22]$} \\
\hline$f_{\mathrm{opt}, 0}$ & $\frac{-2 \beta_{\mathrm{c}}}{2 \alpha_{\mathrm{c}}+5 \beta_{\mathrm{c}}+4 \kappa_{\mathrm{t}}+2 \beta_{\mathrm{c}} \kappa_{\mathrm{t}}-2}$ & {$[-0.45,-0.34]$} \\
$n_{\mathrm{opt}, 0}$ & $\frac{2-2 \beta_{\mathrm{c}}-4 \kappa_{\mathrm{t}}-2 \alpha_{\mathrm{c}}}{2 \alpha_{\mathrm{c}}+5 \beta_{\mathrm{c}}+4 \kappa_{\mathrm{t}}+2 \beta_{\mathrm{c}} \kappa_{\mathrm{t}}-2}$ & {$[-0.48,-0.41]$} \\
\hline$B_{\mathrm{pk}}$ & $\frac{2 \alpha_{\mathrm{c}}+4 \kappa_{\mathrm{t}}-2}{2 \alpha_{\mathrm{c}}+5 \beta_{\mathrm{c}}+4 \kappa_{\mathrm{t}}+2 \beta_{\mathrm{c}} \kappa_{\mathrm{t}}-2}$ & {$[-0.05,+0.14]$} \\
$J_{\mathrm{RMS}}$ & $\frac{\beta_{\mathrm{c}}\left(2 \kappa_{\mathrm{t}}-1\right)}{2 \alpha_{\mathrm{c}}+5 \beta_{\mathrm{c}}+4 \kappa_{\mathrm{t}}+2 \beta_{\mathrm{c}} \kappa_{\mathrm{t}}-2}$ & {$[-0.32,-0.17]$} \\
$r_{\mathrm{W}}$ & 0 & {$[+0.00,+0.00]$} \\
$r_{\mathrm{cw}}$ & 0 & {$[+0.00,+0.00]$} \\
\hline \hline
\end{tabular}

TABLE V

MFT Parameters CF., Fig. 2

\begin{tabular}{|c|c|}
\hline Parameter & Value \\
\hline \multirow[t]{2}{*}{ General } & $\cos (\varphi)=0.85 / V_{\mathrm{RMS}}=600 \mathrm{~V}$ \\
\hline & $x_{\mathrm{cw}}=1.5 / x_{\mathrm{c}}=1.5 / x_{\mathrm{W}}=5.0$ \\
\hline \multirow[t]{3}{*}{ Core } & "TDK N97" \\
\hline & $k_{\mathrm{c}}=1.35 / \alpha_{\mathrm{c}}=1.44 / \beta_{\mathrm{c}}=2.46$ \\
\hline & $f_{\mathrm{c}, \max }=700 \mathrm{kHz} / B_{\mathrm{sat}}=300 \mathrm{mT}$ \\
\hline \multirow[t]{3}{*}{ Windings } & Litz wire \\
\hline & $k_{\mathrm{W}}=25 \% / d_{\mathrm{s}}=100 \mu \mathrm{m} / \sigma=46 \mathrm{MS} / \mathrm{m}$ \\
\hline & $J_{\mathrm{RMS}, \max }=8.0 \mathrm{~A} / \mathrm{mm}^{2}$ \\
\hline \multirow[t]{2}{*}{ Thermal } & $k_{\mathrm{t}}=12.0 / v_{\mathrm{t}}=+0.09 / \kappa_{\mathrm{t}}=-0.11$ \\
\hline & $\Delta T_{\max }=100{ }^{\circ} \mathrm{C} / 40{ }^{\circ} \mathrm{C}$ ambient \\
\hline
\end{tabular}

from $\left.f_{\text {opt. } 0}\right)$ and for designs at the saturation limit. However, for obtaining simple scaling coefficients (cf., (37) and (38)), additional assumptions on $r_{\mathrm{w}}$ and/or $r_{\mathrm{cw}}$ are necessary [34]. Furthermore, it is difficult to draw general conclusions from scaling laws with sub-optimal designs. Therefore, only the scaling of optimal designs $\left(f_{\mathrm{opt}, 0}\right.$ and $\left.n_{\mathrm{opt}, 0}\right)$ is considered in the following.

\section{Analytical Results}

In this section, the aforementioned optimum, frequency diversity, and scaling laws are applied to a specific MFT design. The reference design features the following ratings: $P$ $=20 \mathrm{~kW}, \rho=20 \mathrm{~kW} / \mathrm{L}$, and $V_{\text {RMS }}=600 \mathrm{~V}$.

\section{A. Considered Parameters}

An E-core based MFT with shell-type arrangement is considered (cf., Fig. 2). The key parameters are summarized in Table V. A "TDK N97" ferrite core is selected where the 


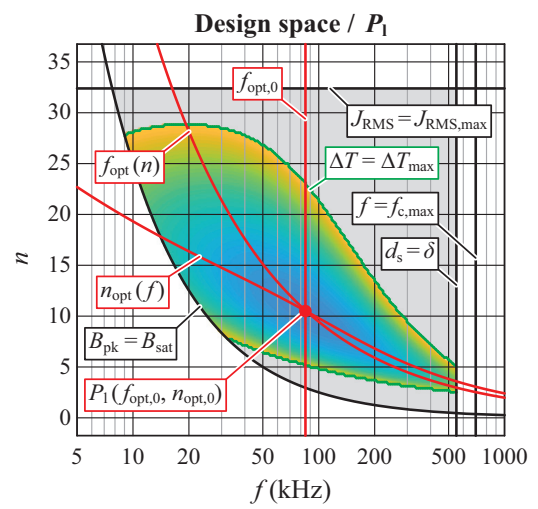

(a)

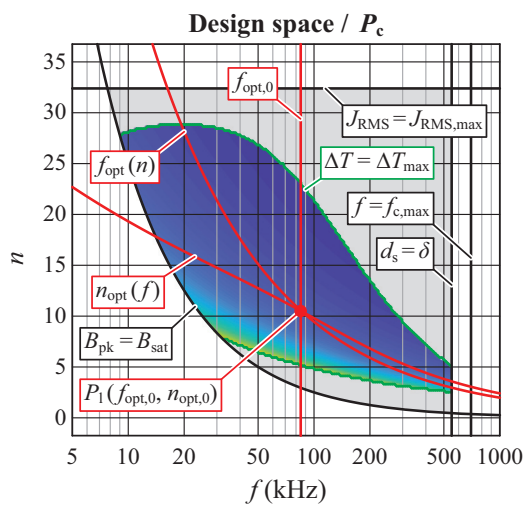

(b)

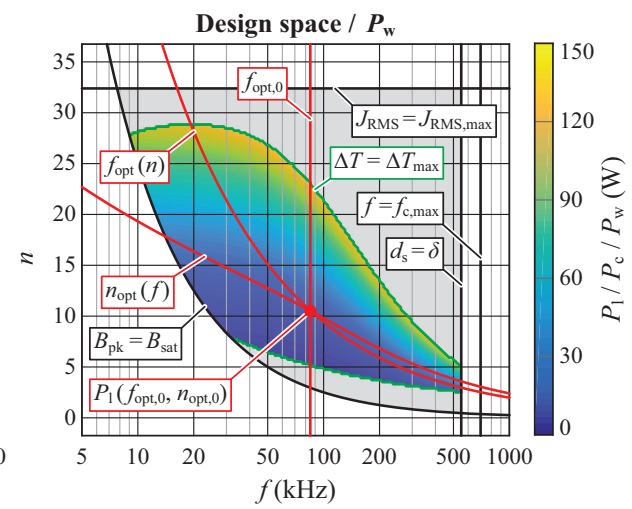

(c)

Fig. 6. Design space of a $20 \mathrm{~kW}$ and $20 \mathrm{~kW} / \mathrm{L}$ MFT (cf., Table V) for different frequencies and numbers of turns. (a) The total losses. (b) The core losses. (c) The winding losses. The design space limits (black and gray), the thermal limit (green), and the optimal designs (red) are highlighted, as shown, schematically, in Fig. 3 (cf., Section III-B).

Steinmetz parameters are extracted with a least square fit of the losses $\left(T=70^{\circ} \mathrm{C}, B_{\mathrm{pk}} \in[25,150] \mathrm{mT}\right.$, and $\left.f \in[50,200] \mathrm{kHz}\right)$ [31]. The maximum error between the considered Steinmetz model and the datasheet values is, in the considered range, less than $5 \%$. The windings are composed of litz wires with $100 \mu \mathrm{m}$ strands. The filling factor of $25 \%$ considers the filling factor of the litz wire itself, the filling factor of the turns, and the insulation distances to the core. The convection coefficient parameters are fitted for the chosen geometry with an air speed of $3 \mathrm{~m} / \mathrm{s}$ with the formula presented in [8].

\section{B. Optimal Design}

Fig. 6(a) depicts the obtained design space (cf., Fig. 3) for $P=20 \mathrm{~kW}$ and $\rho=20 \mathrm{~kW} / \mathrm{L}$. The core losses are diminishing with increasing number of turns and/or frequencies (volt-second product), as shown in Fig. 6(b). On the contrary, the winding losses are increasing with increasing number of turns (current density) and/or frequencies (proximity effect), as shown Fig. 6(c). Therefore, an optimum exists and has the following characteristics: $f_{\mathrm{opt}, 0}=86 \mathrm{kHz}, n_{\mathrm{opt}, 0}=10.5, r_{\mathrm{w}}=1.71, r_{\mathrm{cw}}=$ $0.81, J_{\mathrm{RMS}}=2.9 \mathrm{~A} / \mathrm{mm}^{2}, B_{\mathrm{pk}}=84 \mathrm{mT}, \Delta T=41^{\circ} \mathrm{C}, \eta_{\mathrm{pl}}=99.72 \%$, and $\eta_{\mathrm{fl}}=99.76 \%$. It can be seen that the optimal design is located far away from the design space limits (saturation flux, maximum current density, maximum frequency, and thermal limit).

\section{Frequency Diversity}

Fig. 7(a) shows the design space for $P=20 \mathrm{~kW}$ and different power densities and frequencies. The optimal number of turns ( $n_{\text {opt }}$, as long the saturation flux is not reached) is considered for all the designs. It can be seen that all the optimal designs $\left(f_{\text {opt }, 0}\right)$ are not restricted by the saturation flux, maximum current density, or maximum frequency. This means that the optimum derived in Section III-B can be used. The efficiency is rapidly dropping for designs operated at the saturation flux (cf., Fig. 4).

The frequency diversity presented in Section III-C can be observed in Fig. 7(b) where the Pareto fronts are shown for $f<$

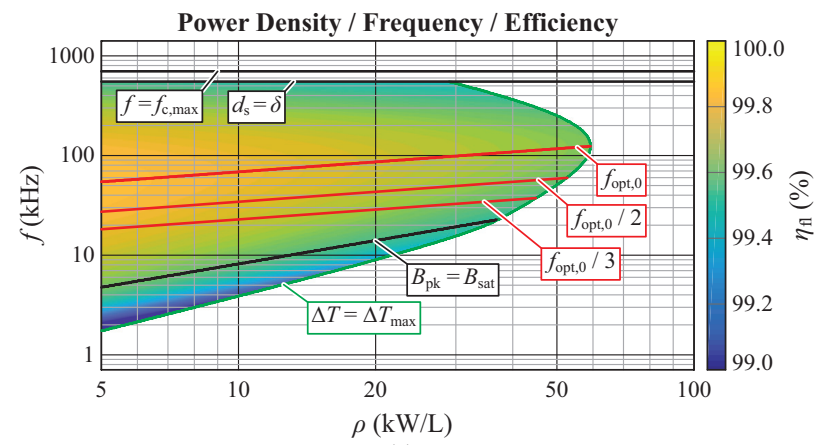

(a)

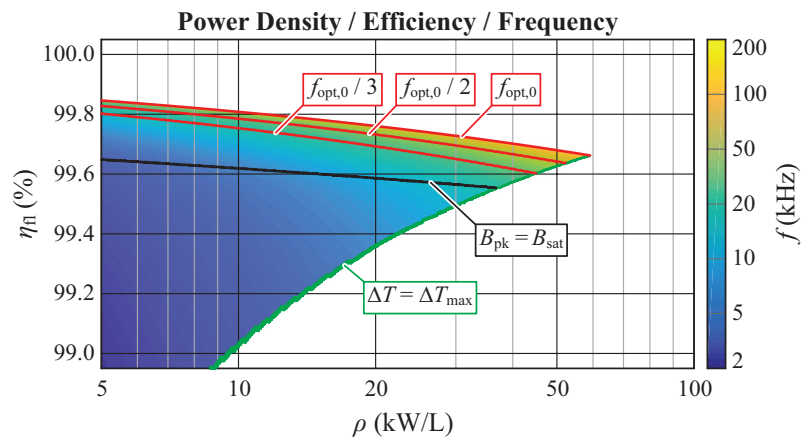

(b)

Fig. 7. (a) Design space for different frequencies and power densities. (b) Pareto front for $f<f_{\text {opt }, 0}$. The design space limits (black), the thermal limit (green), and the optimal designs (red) are shown. The optimal number of turns ( $n_{\mathrm{opt}}$, as long the saturation flux is not reached) is considered for all the designs. The power rating is fixed to $20 \mathrm{~kW}$.

$f_{\text {opt }, 0}$. As expected (cf., Fig. 4(b)), frequencies well below $f_{\text {opt }, 0}$ can be chosen with a minor impact on the efficiency: $\varepsilon=11 \%$ for $\xi=2$ and $\varepsilon=26 \%$ for $\xi=3$ (cf., (36)). It should be noted that, with the considered degrees of freedom (volume, number of turns, and frequency), only a single design (with $f_{\mathrm{opt}, 0}$ and $n_{\mathrm{opt}, 0}$ ) exists at the maximum allowable power density, explaining the sharp tip of the Pareto front.

\section{Scaling Laws}

Fig. 8 depicts the scaling of the aforementioned MFT for different power ratings and power densities (cf., Section III-D). 


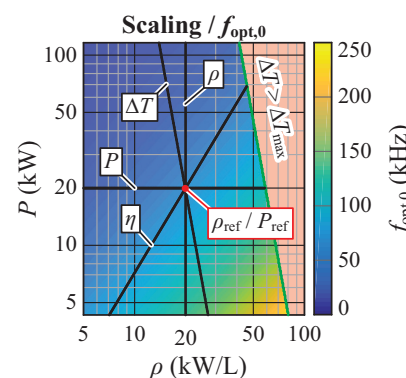

(a)

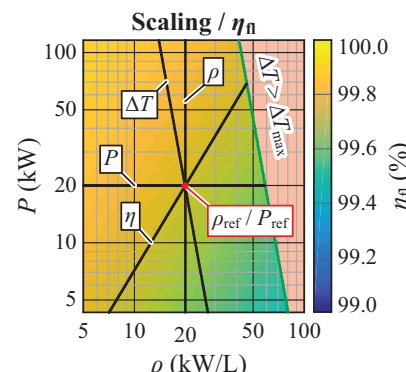

(c)

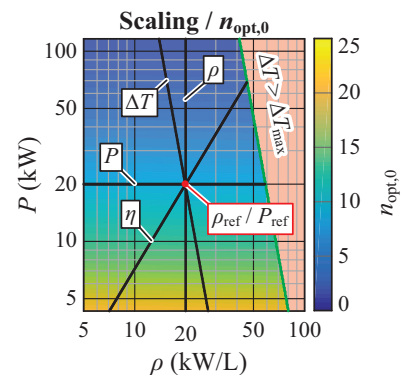

(b)

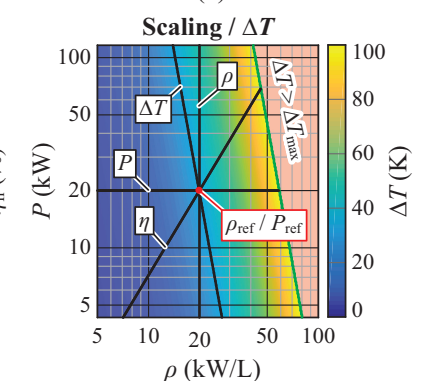

(d)
Fig. 8. Scaling of MFTs for different power ratings $(P)$ and power densities $(\rho)$. (a) Scaling of the frequency. (b) Scaling of the number of turns. (c) Scaling of the efficiency. (d) Scaling of the temperature rise. All the designs are operated with the optimal frequency and the optimal number of turns $\left(f_{\text {opt }, 0}\right.$ and $\left.n_{\text {opt }, 0}\right)$. The different scaling curves are explained, schematically, in Fig. 5 (cf., Section III-D).

All the designs are operated with the optimal frequency and the optimal number of turns $\left(f_{\mathrm{opt}, 0}\right.$ and $\left.n_{\mathrm{opt}, 0}\right)$. The scaling trajectories are shown for constant power rating, constant power density, constant temperature rise, and constant efficiency around the reference design: $P_{\text {ref }}=20 \mathrm{~kW}$ and $\rho_{\text {ref }}=20 \mathrm{~kW} / \mathrm{L}$.

The general conclusions drawn in Section III-D are verified: the compact designs are operated at higher frequencies, but have reduced efficiencies. High power designs are featuring higher efficiencies and reduced frequencies but also limited cooling capabilities.

\section{E. Core Material and Winding Stranding}

The Pareto front obtained with the defined parameters (cf., Fig. 7(b)) can be pushed by changing the core material and the stranding of the windings (all the other parameters remains unchanged, cf., Table V). The following core materials are considered: a low performance ferrite ("TDK N27"), a high performance ferrite ("TDK N97"), a nanocrystalline material ("VAC VITROPERM 500F"), and an amorphous material ("Metglas 2605SA1") [31], [37]-[39]. The strand diameter is chosen between $50 \mu \mathrm{m}, 100 \mu \mathrm{m}$, and $200 \mu \mathrm{m}$. All the designs are operated with the optimal frequency and the optimal number of turns $\left(f_{\mathrm{opt}, 0}\right.$ and $\left.n_{\mathrm{opt}, 0}\right)$.

Fig. 9 shows the obtained Pareto fronts with the corresponding optimal operating frequencies for $P=20 \mathrm{~kW}$. It should be noted that all the designs (until the thermal limit) are not limited by the saturation flux, maximum current density, and maximum frequency. Logically, the designs with fine strands are operated at higher frequencies (cf., (32)). The designs with the ferrite materials feature comparable operating frequencies $\left(\beta_{\mathrm{c}}-\alpha_{\mathrm{c}}\right.$

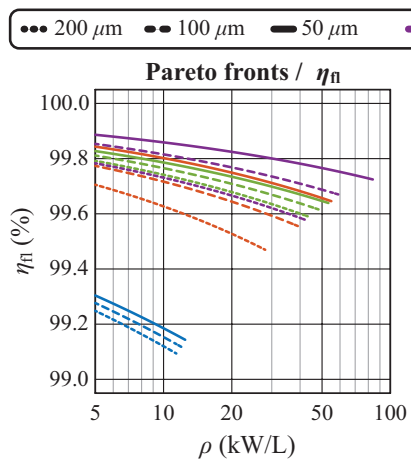

(a)

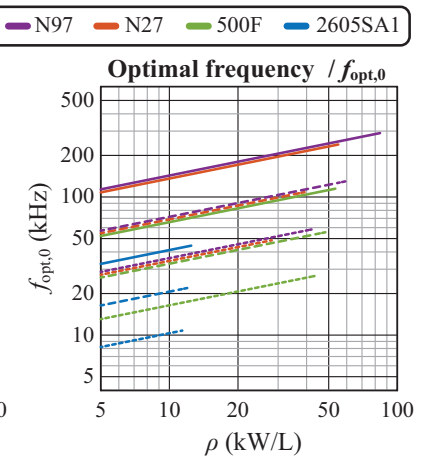

(b)
Fig. 9. (a) Pareto fronts. (b) Operating frequencies for different core materials and winding strandings. All the designs are operated with the optimal frequency and the optimal number of turns $\left(f_{\mathrm{opt}, 0}\right.$ and $\left.n_{\mathrm{opt}, 0}\right)$. The power rating is fixed to $20 \mathrm{~kW}$.

difference is similar, cf., (32)). However, the designs based on the N97 ferrite material are more efficient compared to the designs using the N27 ferrite. The designs with an amorphous core feature low operating frequencies but also low efficiencies. The MFTs with a nanocrystalline core feature high efficiencies with reduced operating frequencies $\left(\beta_{c}-\alpha_{c}\right.$ difference is small) and, therefore, represent an interesting alternative.

It can be concluded that the choice of the core material and the winding stranding are interconnected. Designs with large $\beta_{\mathrm{c}}-\alpha_{\mathrm{c}}$ differences and reduced $d_{\mathrm{s}}$ are operated at higher frequencies (cf., (32)) and feature a reduced $B_{\mathrm{pk}} J_{\mathrm{RMS}}$ product (cf., (24)). However, high optimal operating frequencies do not directly imply high efficiencies. This last statement is highlighted by comparing the N27 ferrite and the nanocrystalline materials in Fig. 9.

\section{NuMERICAL VERIFICATION}

The full-analytical model presented in Section II and the optimum derived in Section III are subject to several simplifications, which are required to obtain an explicit solution for the optimum and the associated scaling laws. In this section, the results obtained with the full-analytical model (cf., Section IV) are compared with a more elaborate seminumerical model in order to verify the validity of the presented results.

\section{A. Semi-Numerical Model}

A semi-numerical model of MFTs, which is based on the analysis presented in [5], [15], [26], [29], is considered. The following effects and limitations are taken into account:

- Geometry: A discrete (integer) number of turns is considered with the exact packing pattern of the turns. The insulation distances (between the turns and to the core) are taken into account.

- Core losses: The flux density, frequency, and temperature dependences of the core losses are considered with a loss map extracted from measurements [15], [16].

- Winding losses: The winding losses are computed with 
the exact packing pattern of the turns and the temperature dependence is taken into account [15], [29].

- Thermal model: A complex thermal model is used where the heat conduction, natural convection, forced convection, and radiation mechanisms are considered. The cross-coupling between the losses and the temperature distribution is taken into account [8], [26], [29], [35]. For the temperature rise, the hotspot of the transformer (typically the winding temperature) is considered.

- Insulation design: The stress in the insulation (electric field and dielectric losses) is computed [21]. Designs with too large electric field values are excluded.

- Equivalent circuit: The effect of the magnetizing and leakage inductances on the applied waveforms is also taken into account [13].

- Parasitic resonances: The effect of the open-circuit and shortcircuit resonances on the MFT operation is investigated [8], [43]. Designs with critically low resonance frequencies are excluded.

This semi-numerical model has been successfully used and experimentally verified with the prototypes presented in [13], [43]-[45]. More particularly, in [45], the semi-numerical model is compared with FEM simulations, electrical measurements, and calorimetric measurements. The deviation between the computed and measured inefficiencies is below $0.05 \%$.

This semi-numerical model is based on analytical equations, but does not feature an analytical solution. Most important, the computation cost for a single design is reasonable: less than 5kFLOPs [46]. Nevertheless, such a model leads to a multivariable, multi-constrained, mixed-integer, and non-linear optimization problem. Therefore, the optimization problem should be solved with a numerical solver. In the following, the computations methods listed below are compared:

- Full-analytical: The presented full-analytical model (cf., Section II) is considered. The derived analytical optimum and scaling laws (cf., Section III) are used.

- Semi-numerical/fixed ratios: The semi-numerical method is used with fixed (constant) geometrical aspect ratios ( $x_{\mathrm{cw}}$, $x_{\mathrm{c}}$, and $x_{\mathrm{w}}$ ).

- Semi-numerical/free ratios: The semi-numerical method is used and the geometrical aspect ratios of the MFT $\left(x_{\mathrm{cw}}, x_{\mathrm{c}}\right.$, and $x_{\mathrm{w}}$ ) are also optimized.

\section{B. Scaling Laws}

The specifications considered in Section IV-A (cf., Table V) are selected and the methods defined in Section V-A are compared. The reference design for the scaling is featuring the same ratings as defined in Section IV-D: $P_{\text {ref }}=20 \mathrm{~kW}$ and $\rho_{\text {ref }}$ $=20 \mathrm{~kW} / \mathrm{L}$. The scaling laws with constant power density $(\rho$ $=$ const. $)$ and constant power $(P=$ const. $)$ are considered since they cover a representative part of the designs shown in Fig. 8.

For the full-analytical model, the derived optimum is used $\left(f_{\mathrm{opt}, 0}\right.$ and $\left.n_{\mathrm{opt}, 0}\right)$. For the two semi-numerical models, the optimum design (with respect to the efficiency) is selected
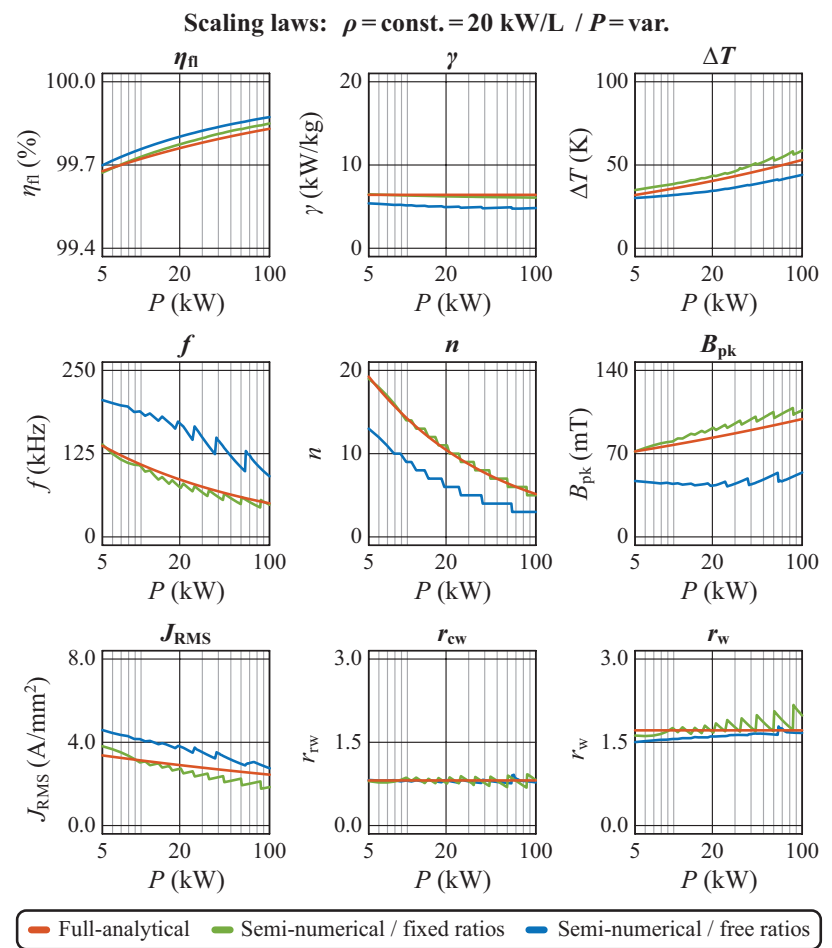

Fig. 10. Scaling for $\rho=$ const. $=20 \mathrm{~kW} / \mathrm{L}$. The presented scaling laws (fullanalytical model, cf., Section IV) are compared to semi-numerical models with fixed and free geometrical aspect ratios.

with a mixed-integer multi-constrained genetic algorithm [27], [47]. The results are shown in Fig. 10 and Fig. 11 for constant power density ( $\rho=$ const.) and constant power $(P=$ const.), respectively.

It can be seen immediately that the consideration of discrete (integer) numbers of turns leads to discontinuous curves for the optimal designs. The derived scaling laws are valid as they correctly predict (qualitatively and quantitatively) the trends for all the considered parameters for fixed geometrical aspect ratios. With free geometrical aspect ratios, the optimal designs feature narrow winding windows and, therefore, reduced proximity effect losses (cf., (10)) and higher switching frequencies (cf., (32)). The increased switching frequencies also explain the reduced numbers of turns and the low flux densities. However, the thickness of the winding window cannot be reduced beyond a certain limit, due to practical constraints (packing of the turns) and due to the incompressible insulation distances (e.g., coil former thickness). Nevertheless, the optimization of the geometrical aspect ratios only provides limited advantages for the achieved efficiencies and power densities. This confirms the aspect ratios can be kept constant for the analysis of the fundamental characteristics of MFTs with an analytical model (i.e., scaling laws).

High efficiencies (up to 99.85\%) and power densities (up to $63 \mathrm{~kW} / \mathrm{L}$ ) are achieved for the selected parameters. However, it should be noted that the presented designs are based on ideal characteristics: every core shape and every litz wire (number of strands and shape) is considered as available. The 

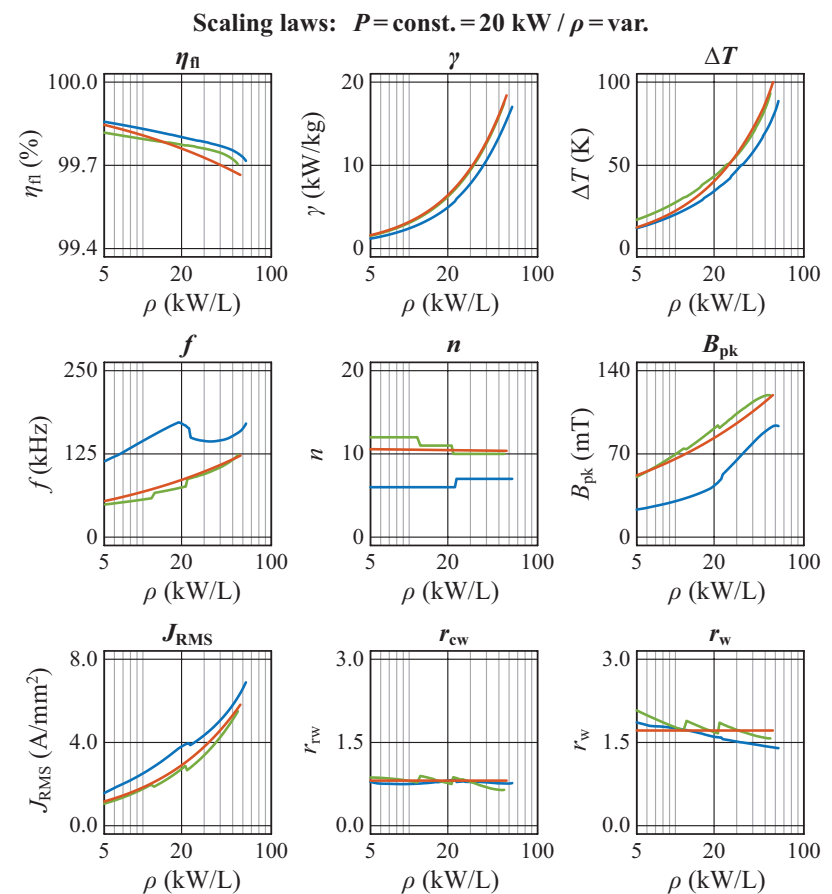

- Full-analytical - Semi-numerical / fixed ratios - Semi-numerical / free ratios

Fig. 11. Scaling for $P=$ const. $=20 \mathrm{~kW}$. The presented scaling laws (fullanalytical model, cf., Section IV) are compared to semi-numerical models with fixed and free geometrical aspect ratios.

thermal model assumes a uniform forced convection cooling from all the sides $\left(40^{\circ} \mathrm{C}\right.$ ambient). Moreover, the volume and the losses of the cooling system (e.g., fans) are not taken into account. Finally, only the MFT is optimized without considering external factors (e.g., switching losses of the power semiconductors). Therefore, the performances shown in Figs. 10 and 11 are unlikely to be achieved for a constructed prototype and represent the theoretical limit. Nevertheless, such practical design restrictions could be easily integrated into the aforementioned computation methods.

The optimal designs obtained with the three considered models feature similar efficiencies but different parameters, especially for the semi-numerical model with free geometrical aspect ratios. More particularly, large deviations can be observed for the operating frequency, the number of turns, and, therefore, the flux density. The discrepancies between the models and the underlying design space diversity are analyzed in more details in the next subsection.

\section{Design Space Diversity}

The parameter ranges defined in Section IV-A (cf., Table V) are selected with the following nominal ratings: $P=20 \mathrm{~kW}$ and $\rho$ $=20 \mathrm{~kW} / \mathrm{L}$. The complete design space is swept for the three methods defined in Section V-A. A brute-force algorithm is used in order to explore the complete design space including eventual local minima. For each method, all the designs, which feature less than $15 \%$ more losses than the optimal design are kept. Fig. 12(a) shows the results obtained with the full-

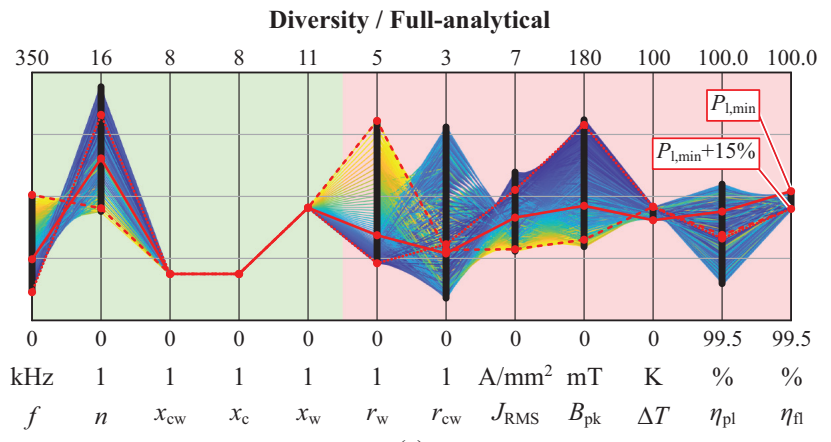

(a)

Diversity / Semi-numerical / Fixed ratios

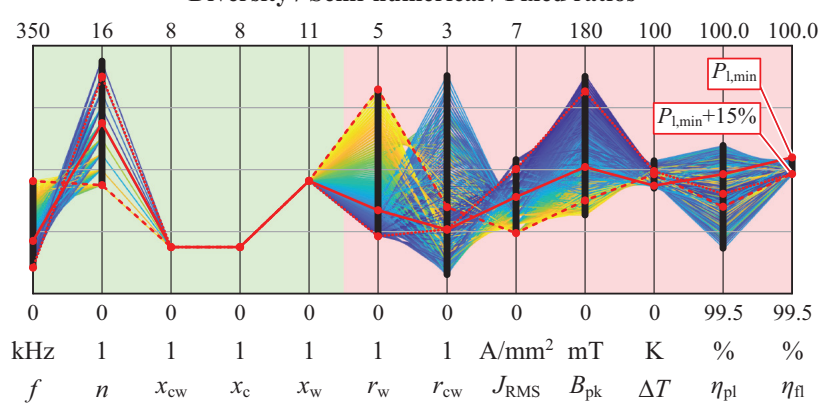

(b)

Diversity / Semi-numerical / Free ratios

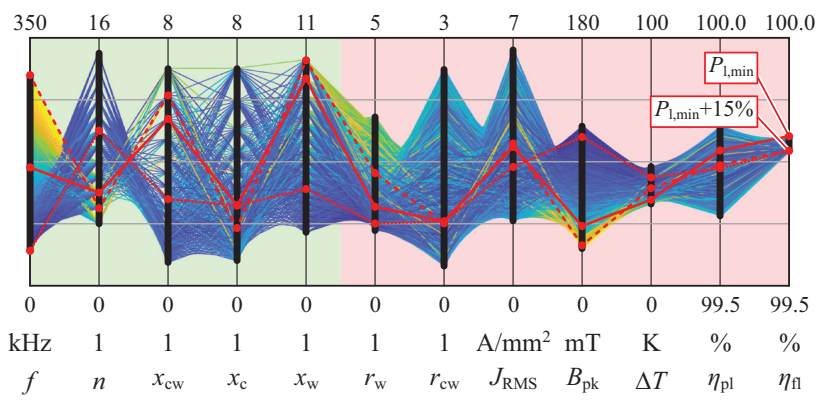

(c)

- Optimal ...... Min. freq. $\cdots$ Max. freq. — Var. range Input var. $\square$ Output var.

Fig. 12. Parallel coordinate plots for $P=$ const. $=20 \mathrm{~kW}$ and $\rho=20 \mathrm{~kW} / \mathrm{L}$. (a) The full-analytical model (1200 designs). (b) The semi-numerical model with fixed geometrical aspect ratios (500 designs). (c) The semi-numerical model with free geometrical aspect (300000 designs). All the designs, which feature less than $15 \%$ more losses than the optimal design are shown. The designs with the optimal, minimum, and maximum frequencies are highlighted in red.

analytical model, Fig. 12(b) shows the results obtained with the semi-numerical model with fixed geometrical aspect ratios, and, finally, Fig. 12(c) depicts the results obtained with the semi-numerical model with free geometrical aspect ratios. The results are displayed with parallel coordinate plots, where the designs with the optimal, minimum, and maximum frequencies are highlighted [48].

The full-analytical model delivers results similar to the semi-numerical model for fixed geometrical aspect ratios. A significant frequency diversity can be observed (cf., Section III-C). The designs with high operating frequencies feature low numbers of turns, low flux densities, and low current densities. The design space diversity is even greater if the geometrical aspect ratios are additionally optimized. For this last case, the 
TABLE VI

Design Space Diversity / Ranges / CF., Fig. 12(C)

\begin{tabular}{lc|lc}
\hline \hline Var. & Range & Var. & Range \\
\hline$P$ & $20 \mathrm{~kW}$ & $\rho$ & $20 \mathrm{~kW} / \mathrm{L}$ \\
$f$ & {$[49,297] \mathrm{kHz}$} & $r_{\mathrm{W}}$ & {$[1.11,3.40]$} \\
$n$ & {$[4,15]$} & $r_{\mathrm{cW}}$ & {$[0.24,2.60]$} \\
$x_{\mathrm{cW}}$ & {$[0.75,7.00]$} & $J_{\mathrm{RMS}}$ & {$[1.8,6.7] \mathrm{A} / \mathrm{mm}^{2}$} \\
$x_{\mathrm{c}}$ & {$[0.81,7.00]$} & $B_{\mathrm{pk}}$ & {$[27,116] \mathrm{mT}$} \\
$x_{\mathrm{W}}$ & {$[2.37,10.00]$} & $\Delta T$ & {$[33,48] \mathrm{K}$} \\
$\eta_{\mathrm{pl}}$ & {$[99.64,99.82] \%$} & $\eta_{\mathrm{fl}}$ & {$[99.77,99.80] \%$} \\
\hline \hline
\end{tabular}

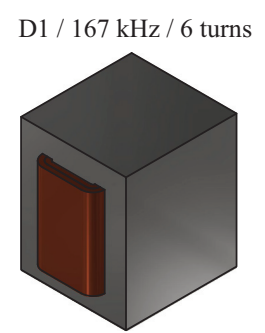

D4 / $159 \mathrm{kHz} / 8$ turns

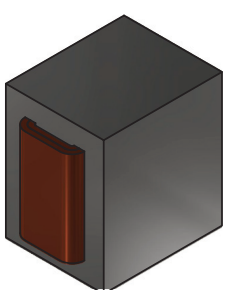

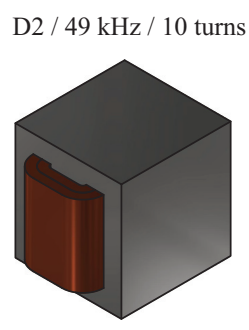

D5 / $161 \mathrm{kHz} / 5$ turns

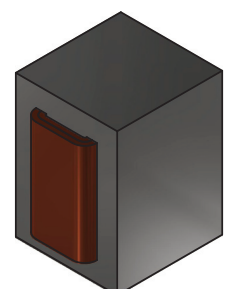

D3 / $297 \mathrm{kHz} / 5$ turns

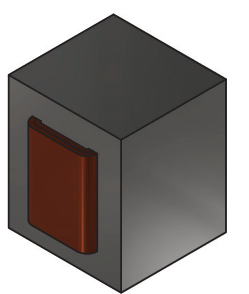

D6 / $77 \mathrm{kHz} / 6$ turns

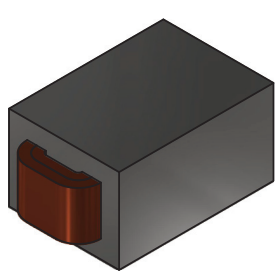

Fig. 13. Selected designs (20 kW and $20 \mathrm{~kW} / \mathrm{L}$, cf., Fig. 12(c)) with the following features: minimum losses ("D1"), minimum frequency ("D2"), maximum frequency ("D3"), minimum ratio between core and winding losses ("D4"), maximum ratio between core and winding losses ("D5"), and flat geometrical aspect ratios ("D6"). The parameters of the different designs are described in Table VII.

variables vary in wide ranges, as shows in Table VI.

Some designs, computed with the semi-numerical model (cf., Fig. 12(c)), are highlighted in Fig. 13 and Table VII. The following designs are selected: minimum losses ("D1"), minimum frequency ("D2"), maximum frequency ("D3"), minimum ratio between core and winding losses ("D4"), maximum ratio between core and winding losses ("D5"), and flat geometrical aspect ratios ("D6"). Despite extremely different parameters (e.g., frequency, core losses, winding losses, flux density, and current density) all selected designs feature very similar full-load efficiencies $\left(\eta_{\mathrm{ff}}\right)$. However, it should be noted that, depending on the ratio between the core and winding losses $\left(r_{\mathrm{cw}}\right)$, the partial-load efficiencies $\left(\eta_{\mathrm{pl}}\right)$ are different.

It can be seen that extremely different designs feature similar performances. This diversity would be even greater with additional degrees of freedom (e.g., core material and winding stranding). The design space diversity can be used to select a quasi-optimal design with additional design restrictions (e.g.,
TABLE VII

Design Space Diversity / Designs / CF., Fig. 12(C)

\begin{tabular}{lccccccc}
\hline \hline Var. & Unit & $\mathrm{D} 1$ & $\mathrm{D} 2$ & $\mathrm{D} 3$ & $\mathrm{D} 4$ & $\mathrm{D} 5$ & $\mathrm{D} 6$ \\
\hline$P$ & $\mathrm{~kW}$ & 20 & 20 & 20 & 20 & 20 & 20 \\
$\rho$ & $\mathrm{kW} / \mathrm{L}$ & 20 & 20 & 20 & 20 & 20 & 20 \\
$f$ & $\mathrm{kHz}$ & 167 & 49 & 297 & 159 & 161 & 77 \\
$n$ & & 6 & 10 & 5 & 8 & 5 & 6 \\
$x_{\mathrm{cW}}$ & & 5.38 & 2.79 & 7.00 & 4.72 & 4.14 & 6.14 \\
$x_{\mathrm{c}}$ & & 2.59 & 3.06 & 2.19 & 3.06 & 2.59 & 4.26 \\
$x_{\mathrm{W}}$ & & 9.19 & 4.67 & 10.00 & 9.19 & 10.00 & 2.58 \\
$r_{\mathrm{W}}$ & & 1.59 & 1.23 & 2.12 & 1.59 & 1.62 & 1.71 \\
$r_{\mathrm{cW}}$ & & 0.77 & 0.77 & 0.61 & 0.24 & 2.60 & 0.79 \\
$J_{\mathrm{RMS}}$ & $\mathrm{A} / \mathrm{mm}{ }^{2}$ & 3.9 & 3.3 & 4.4 & 4.6 & 2.7 & 3.0 \\
$\hat{B}$ & $\mathrm{mT}$ & 43 & 106 & 27 & 35 & 59 & 79 \\
$\Delta T$ & $\mathrm{~K}$ & 35 & 43 & 39 & 41 & 37 & 42 \\
$\eta_{\mathrm{pl}}$ & $\%$ & 99.77 & 99.74 & 99.76 & 99.82 & 99.64 & 99.74 \\
$\eta_{\mathrm{fl}}$ & $\%$ & 99.80 & 99.77 & 99.77 & 99.77 & 99.77 & 99.77 \\
\hline \hline
\end{tabular}

available core shapes, available litz wires, and switching losses of the power semiconductors). The systematic exploration of the design space is also useful for drawing general conclusions on the advantages and limitations of the different models.

\section{CRitical Analysis}

With the aforementioned results, different conclusions can be made on the full-analytical modeling (cf., Section II), the corresponding optimum, and the scaling laws (cf., Section III).

- The full-analytical model is able to correctly capture qualitatively and quantitatively the design process of MFTs. However, many effects are neglected. This implies that some designs can be invalid (e.g., due to parasitics and thermal limits), difficult to construct (e.g., turn packing), and/or feature reduced efficiencies (e.g., due to simplified loss models and neglected temperature dependences of the losses).

- For a given core geometry, the optimal design can be analytically extracted. Since the optimum is always flat with respect to frequency, the optimal operating frequency of the converter (considering switching losses of the power semiconductors) can be significantly lower than the optimal frequency of the MFT.

- The analytical optimum of the MFTs should be considered with caution. Extremely different designs (e.g., concerning frequency, flux density, and ratio between core and winding losses) can lead to similar performances (design space diversity). This implies that the designs located around the global minimum (analytical optimum) and around local minima cannot be neglected during the design process. Nevertheless, the analytical optimum is useful to quantitatively understand the fundamental limitations of MFTs.

- An optimal design can be scaled for different power ratings and power densities. These scaling laws correctly predict 
the trends and limitations of MFT designs. However, due to the required assumptions (e.g., fixed core material, fixed winding stranding, and fixed geometrical aspect ratios), these scaling laws are limited to theoretical analyses and cannot be used directly for the complete design process of MFT prototypes.

It appears that the limitations of the full-analytical model are problematic for obtaining an accurate model of MFTs, which is required for virtual prototyping and design automation. Therefore, semi-numerical models should be used and the following modeling and optimization guidelines can be extracted (cf., Section V):

- A multi-variable, multi-constrained, mixed-integer, and nonlinear optimization problem should be solved. Due to the design space diversity, many local minima exist. Moreover, the different local minima are flat, implying that designs located near a minimum can be almost as good as the minimum itself. Therefore, a robust optimization algorithm (e.g., particle swarm and genetic) should be selected [27], [47]. For designs with many discrete variables, a brute-force search algorithm may be the only practicable solution. Additionally, the complete DC-DC converter (e.g., semiconductors and DC-link capacitors) should also be considered for the optimization process of the MFT due to the cross-coupling between the components (mainly through the switching frequency).

- In order to fully utilize the degrees of freedom offered by MFTs, the design space diversity should be checked after the optimization process. An important diversity implies that additional objectives (e.g., partial-load efficiencies, mass, or cost) can be added without any significant impact on the primary goals (e.g., efficiency and volume).

- For a practical MFT design, many additional restrictions apply (e.g., available core shapes, available litz wires, and manufacturability constraints). However, the aforementioned design space diversity mitigates the impact of such design restrictions on the achievable performances.

- Due to the flat optimum, model uncertainties and parameter tolerances can significantly impact the design parameters (e.g., switching frequency, flux density, and current density) of the optimal designs, however, with a minor impact on the achieved performances (e.g., efficiency, volume, and mass) [13].

- The full-analytical model can be used as a filter to reduce the design space (e.g., geometry, core material, and litz wire) before applying the more complex semi-numerical model.

- Models based on numerical field simulations (e.g., FEM simulations) are extremely time-consuming (modeling complexity, and computational cost) and, therefore, are not adapted for optimizations [7], [21]. However, field simulations are useful for checking and fine-tuning the designs extracted during the optimization process.

\section{Conclusion}

This paper investigates the optimization and scaling of MFTs. A simple full-analytical model is presented for the core losses (using the Steinmetz parameters), winding losses (including the HF losses), and temperature rise (convection process). With this model, the design space of MFTs is analyzed and the optimum is derived (frequency and number of turns). An increased operating frequency allows for more efficient and more compact designs. However, beyond a certain frequency, the performance cannot be further improved due to the increased core and HF winding losses. The properties of the optimum are analyzed and it is found that the optimum is always flat: the optimal operating frequency can be divided by two with less than $15 \%$ additional losses (the number of turns is adapted). At the optimum, the flux density is typically lower than the saturation flux density of the core.

Scaling laws are derived for optimal MFTs operated at different power ratings (with constant power density, constant efficiency, or constant temperature rise) and power densities (with constant power rating). These scaling laws allow the extraction of general statements about MFT performances. Compact designs are operated at higher frequencies, but have reduced efficiencies. High power designs are featuring higher efficiencies and reduced frequencies but also limited cooling capabilities.

A $20 \mathrm{~kW}$ and $20 \mathrm{~kW} / \mathrm{L}\left(328 \mathrm{~W} / \mathrm{in}^{3}\right)$ MFT with a ferrite core and litz wire windings is considered for a numerical evaluation. The proposed full-analytical model, optimum, and scaling laws are applied. The obtained results are successfully verified with a more elaborate semi-numerical model. It is found that the full-analytical model correctly predicts the behavior of MFTs. However, due to the multiple local minima of the mapping between the design space and the performance space (design space diversity), the analytical optimum cannot be used for the complete design process of high-performance MFT prototypes. Therefore, global multi-objective optimization should be performed with more advanced semi-numerical models.

\section{ACKNOWLEDGMENT}

The research has been carried out within the frame of the Swiss Competence Center for Energy Research on the Future Swiss Electrical Infrastructure (SCCER-FURIES) with the financial support of the Swiss Innovation Agency. The authors would like to thank Michael Leibl for laying the foundations of this work.

\section{REFERENCES}

[1] S. V. Kulkarni and S. A. Khaparde, Transformer Engineering: Design and Practice. CRC Press, Boca Raton, 2004.

[2] "Verteiltransformatoren (in German)," Deutsches Kupferinstitut, Feb. 2007.

[3] H. Akagi, S. Kinouchi, and Y. Miyazaki, "Bidirectional isolated dualactive-bridge (DAB) DC-DC converters using $1.2 \mathrm{kV} 400 \mathrm{~A} \mathrm{SiC}$ MOSFET dual modules," in CPSS Transactions on Power Electronics 
and Applications, vol. 1, no. 1, pp. 33-40, Dec. 2016.

[4] F. Kieferndorf, U. Drofenik, F. Agostini, and F. Canales, "Modular PET, two-phase air-cooled converter cell design and performance evaluation with 1.7 kV IGBTs for MV applications," in Proceedings of 2016 IEEE Applied Power Electronics Conference and Exposition (APEC), Long Beach, CA, 2016, pp. 472-479.

[5] M. Leibl, G. Ortiz, and J. W. Kolar, "Design and experimental analysis of a medium-frequency transformer for solid-state transformer applications," in IEEE Journal of Emerging and Selected Topics in Power Electronics, vol. 5, no. 1, pp. 110-123, Mar. 2017.

[6] S. Zhao, Q. Li, and F. C. Lee, "High frequency transformer design for modular power conversion from medium voltage AC to $400 \mathrm{~V} \mathrm{DC}$," in Proceedings of 2017 IEEE Applied Power Electronics Conference and Exposition (APEC), Tampa, FL, 2017, pp. 2894-2901.

[7] T. Guillod, F. Krismer, and J. W. Kolar, "Electrical shielding of MV/MF transformers subjected to high $\mathrm{d} v / \mathrm{d} t$ PWM voltages," in Proceedings of 2017 IEEE Applied Power Electronics Conference and Exposition (APEC), Tampa, FL, 2017, pp. 2502-2510.

[8] V. C. Valchev and A. Van den Bossche, Inductors and Transformers for Power Electronics. Taylor \& Francis, Ablingdon, 2005.

[9] J. A. Ferreira, Electromagnetic Modelling of Power Electronic Converters. Springer, Science \& Business Media, Berlin, 2013.

[10] F. C. Schwarz, "A method of resonant current pulse modulation for power converters," in IEEE Transactions on Industrial Electronics and Control Instrumentation, vol. IECI-17, no. 3, pp. 209-221, May 1970.

[11] R. M. Burkart and J. W. Kolar, "Comparative $\eta-\rho-\sigma$ Pareto optimization of $\mathrm{Si}$ and $\mathrm{SiC}$ multilevel dual-active-bridge topologies with wide input voltage range," in IEEE Transactions on Power Electronics, vol. 32, no. 7, pp. 5258-5270, Jul. 2017.

[12] J. W. Kolar, D. Bortis, and D. Neumayr, "The ideal switch is not enough," in Proceedings of 2016 28th International Symposium on Power Semiconductor Devices and ICS (ISPSD), Prague, 2016, pp. $15-22$.

[13] T. Guillod, F. Krismer, and J. W. Kolar, "Magnetic equivalent circuit of MF transformers: modeling and parameters uncertainties," in Springer Electrical Engineering, vol. 100, no. 4, pp. 2261-2275, 2018.

[14] H. Kleinrath, "Ersatzschaltbilder für transformatoren und asynchronmaschinen (in German)," in $e \& i$, vol. 110, no. 1, pp. 68-74, 1993.

[15] J. Mühlethaler, "Modeling and multi-objective optimization of inductive power components," Ph.D. dissertation, ETH Zürich, Zurich, Swiss, 2012.

[16] K. Venkatachalam, C. R. Sullivan, T. Abdallah, and H. Tacca, "Accurate prediction of ferrite core loss with nonsinusoidal waveforms using only Steinmetz parameters," in Proceedings of 2002 IEEE Workshop on Computers in Power Electronics, Mayaguez, Puerto Rico, USA, 2002, pp. 36-41.

[17] A. M. Urling, V. A. Niemela, G. R. Skutt, and T. G. Wilson, "Characterizing high-frequency effects in transformer windings-a guide to several significant articles," in Proceedings of Fourth Annual IEEE Applied Power Electronics Conference and Exposition, Baltimore, MD, USA, 1989, pp. 373-385.

[18] T. Guillod, J. Huber, F. Krismer, and J. W. Kolar, "Litz wire losses: Effects of twisting imperfections," in Proceedings of 2017 IEEE 18th Workshop on Control and Modeling for Power Electronics (COMPEL), Stanford, CA, 2017, pp. 1-8.

[19] W. G. Hurley, E. Gath, and J. G. Breslin, "Optimizing the AC resistance of multilayer transformer windings with arbitrary current waveforms," in IEEE Transactions on Power Electronics, vol. 15, no. 2, pp. 369-376, Mar. 2000.

[20] J. Mühlethaler and J. W. Kolar, "Optimal design of inductive components based on accurate loss and thermal models," in Proceedings of Tutorial at the IEEE Applied Power Electronics Conference and Exposition (APEC), Feb. 2012.

[21] T. Guillod, R. Faerber, D. Rothmund, F. Krismer, C. M. Franck, and J. W. Kolar, "Dielectric losses in dry-type insulation of medium-voltage power electronic converters," in IEEE Journal of Emerging and Selected Topics in Power Electronics (Early Access), May 2019.
[22] M. Leibl, "Three-phase PFC rectifier and high-voltage generator," Ph.D. dissertation, ETH Zürich, Zurich, Swiss, 2017.

[23] W. J. Gu and R. Liu, "A study of volume and weight vs. frequency for high-frequency transformers," in Proceedings of IEEE Power Electronics Specialists Conference (PESC'93), Seattle, WA, USA, 1993, pp. 1123-1129.

[24] W. G. Hurley, W. H. Wölfle, and J. G. Breslin, "Optimized transformer design: inclusive of high-frequency effects," in IEEE Transactions on Power Electronics, vol. 13, no. 4, pp. 651-659, Jul. 1998.

[25] P. Shuai and J. Biela, "Design and optimization of medium frequency, medium voltage transformers," in Proceedings of 2013 15th European Conference on Power Electronics and Applications (EPE), Lille, 2013, pp. 1-10.

[26] M. Mogorovic and D. Dujic, "100 kW, $10 \mathrm{kHz}$ medium-frequency transformer design optimization and experimental verification," in IEEE Transactions on Power Electronics, vol. 34, no. 2, pp. 1696-1708, Feb. 2019.

[27] A. Garcia-Bediaga, I. Villar, A. Rujas, L. Mir, and A. Rufer, "Multiobjective optimization of medium-frequency transformers for isolated soft-switching converters using a genetic algorithm, " in IEEE Transactions on Power Electronics, vol. 32, no. 4, pp. 2995-3006, Apr. 2017.

[28] M. A. Bahmani, T. Thiringer, and M. Kharezy, "Optimization and experimental validation of medium-frequency high power transformers in solid-state transformer applications," in Proceedings of 2016 IEEE Applied Power Electronics Conference and Exposition (APEC), Long Beach, CA, 2016, pp. 3043-3050.

[29] I. Villar, A. Garcia-Bediaga, U. Viscarret, I. Etxeberria-Otadui, andA. Rufer, "Proposal and validation of medium-frequency power transformer design methodology," in Proceedings of 2011 IEEE Energy Conversion Congress and Exposition (ECCE USA), Phoenix, AZ, 2011, pp. 3792 3799.

[30] Grain Oriented Electrical Steel Powercore, ThyssenKrupp, Essen, May 2017.

[31] SIFERRIT Material N97, TDK, Tokyo, May 2017.

[32] M. Mogorovic and D. Dujic, "Sensitivity analysis of medium frequency transformer design," in Proceedings of 2018 International Power Electronics Conference (IPEC-Niigata 2018-ECCE Asia), Niigata, 2018, pp. 2170-2175.

[33] W. G. Hurley, T. Merkin, and M. Duffy, "The performance factor for magnetic materials revisited: the effect of core losses on the selection of core size in transformers," in IEEE Power Electronics Magazine, vol. 5, no. 3, pp. 26-34, Sep. 2018.

[34] W. G. Odendaal and J. A. Ferreira, "Effects of scaling high-frequency transformer parameters," in IEEE Transactions on Industry Applications, vol. 35, no. 4, pp.932-940, Jul.-Aug. 1999.

[35] R. Burkart, "Advanced modeling and multi-objective optimization of power electronic converter systems," Ph.D. dissertation, ETH Zürich, Zurich, Swiss, 2016.

[36] M. Mogorovic and D. Dujic, "Thermal modeling and experimental verification of an air cooled medium frequency transformer," in Proceedings of 2017 19th European Conference on Power Electronics and Application (EPE'17 ECCE Europe), Warsaw, 2017, pp. 1-9.

[37] VITROPERM 500F / VITROVAC 6030F, Vacuumschmelze, Hanau, Apr. 2003.

[38] Magnetic Alloy 2605SA1 (Iron-Based), Metglas, Conway, SC, Jun. 2011.

[39] SIFERRIT Material N27, TDK, Tokyo, May 2017.

[40] SIFERRIT Material N49, TDK, Tokyo, May 2017.

[41] SIFERRIT Material N87, TDK, Tokyo, Sep. 2017.

[42] SIFERRIT Material N95, TDK, Tokyo, May 2017.

[43] D. Rothmund, G. Ortiz, T. Guillod, and J. W. Kolar, "10 kV SiC-based isolated DC-DC converter for medium voltage-connected solid-state transformers," in Proceedings of 2015 IEEE Applied Power Electronics Conference and Exposition (APEC), Charlotte, NC, 2015, pp. 10961103.

[44] D. Neumayr, M. Vöhringer, N. Chrysogelos, G. Deboy, and J. W. Kolar, "P ${ }^{3}$ DCT-partial-power pre-regulated DC transformer," in IEEE Transactions on Power Electronics, vol. 34, no. 7, pp. 6036-6047, Jul. 2019.

[45] T. Guillod, "Modeling and design of medium-frequency transformers for future medium-voltage power electronics interfaces," Ph.D. dissertation, 
ETH Zürich, Zurich, Swiss, 2018.

[46] A. Quarteroni and F. Saleri, Scientific Computing with MATLAB. Springer, Berlin, Heidelberg, 2004.

[47] Global Optimization Toolbox-User's Guide, MathWorks, Natick, MA, Mar. 2019.

[48] J. Yang, W. Peng, M. O. Ward, and E. A. Rundensteiner, "Interactive hierarchical dimension ordering, spacing and filtering for exploration of high dimensional datasets," in Proceedings of IEEE Symposium on Information Visualization 2003 (IEEE Cat. No.03TH8714), Seattle, WA, USA, 2003, pp. 105-112.

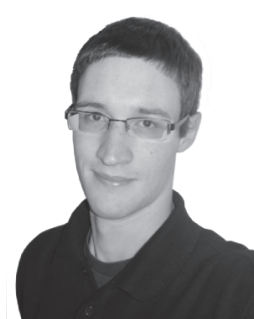

Thomas Guillod received the M.Sc. degree in electrical engineering and information technology in 2013 from ETH Zurich, Switzerland with a focus on power electronics, numerical analysis, and field theory. In 2013, he joined the Power Electronic Systems Laboratory at ETH Zurich as a Ph.D. student and, in 2018, as a postdoctoral researcher. His current research interests include MV converters design, MF transformer optimization, electrical insulation in power converters, and numerical methods.

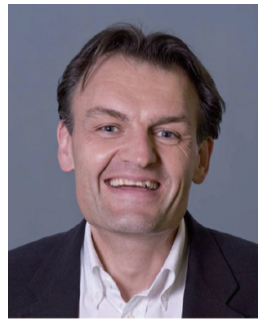

Johann Walter Kolar received his M.Sc. and Ph.D. degrees (summa cum laude/promotio sub auspiciis praesidentis rei publicae) from the University of Technology Vienna, Austria, in 1997 and 1999, respectively. Since 1984, he has been working as an Independent Researcher and International Consultant in close collaboration with the University of Technology Vienna, in the fields of power electronics, industrial electronics and high performance drive systems. He has proposed numerous novel PWM converter topologies, modulation and control concepts and has supervised 70+ Ph.D. students. He has published $880+$ scientific papers in international journals and conference proceedings, 4 book chapters, and has filed $190+$ patents. The focus of his current research is on ultra-compact and ultra-efficient $\mathrm{SiC}$ and $\mathrm{GaN}$ converter systems, solid-state transformers, advanced variable speed three-phase motor drives, integrated modular motor drives, ultra-high speed motors, bearingless motors/ actuators, and design automation in power electronics/mechatronics. Dr. Kolar has received 27 IEEE Transactions and Conference Prize Paper Awards, the 2014 IEEE Middlebrook Award, the 2016 IEEE William E. Newell Power Electronics Award, the 2016 IEEE PEMC Council Award and two ETH Zurich Golden Owl Awards for excellence in teaching. He initiated and/or is the founder of four ETH Spin-off companies. He is a member of the steering committees of several leading international conferences in the field and has served from 2001 through 2013 as an associate editor of the IEEE Transactions on Power Electronics. Since 2002 he also is an associate editor of the Journal of Power Electronics of the Korean Institute of Power Electronics and a member of the Editorial Advisory Board of the IEEJ Transactions on Electrical and Electronic Engineering. 